\title{
gु \\ Termination of typical wave-function multifractal spectra at the Anderson metal-insulator transition: Field theory description using the functional renormalization group
}

\author{
Matthew S. Foster, ${ }^{1, *}$ Shinsei Ryu, ${ }^{2}$ and Andreas W. W. Ludwig ${ }^{3}$ \\ ${ }^{1}$ Department of Physics, Columbia University, New York, New York 10027, USA and \\ Department of Physics and Astronomy, Rutgers University, Piscataway, New Jersey 08854, USA \\ ${ }^{2}$ Department of Physics, University of California, Berkeley, California 94720, USA \\ ${ }^{3}$ Department of Physics, University of California, Santa Barbara, California 93106, USA \\ (Received 4 February 2009; revised manuscript received 15 June 2009; published 3 August 2009)
}

\begin{abstract}
We revisit the problem of wave-function statistics at the Anderson metal-insulator transition (MIT) of noninteracting electrons in $d>2$ spatial dimensions. At the transition, the complex spatial structure of the critical wave functions is reflected in the nonlinear behavior of the multifractal spectrum of generalized inverse participation ratios (IPRs). Beyond the crossover from narrow to broad IPR statistics, which always occurs for sufficiently large moments of the wave-function amplitude, the spectrum obtained from a typical wave function associated with a particular disorder realization differs markedly from that obtained from the disorderaveraged IPRs. This phenomenon is known as the termination of the multifractal spectrum. We provide a field theoretical derivation for the termination of the typical multifractal spectrum by combining the nonlinear sigma model framework, conventionally used to access the MIT in $d=2+\epsilon$ dimensions, with a functional renormalization-group (FRG) technique. The FRG method deployed here was originally pioneered to study the properties of the two-dimensional (2D) random-phase $X Y$ model [D. Carpentier and P. Le Doussal, Nucl. Phys. B 588, 565 (2000)]. The same method was used to demonstrate the termination of the multifractal spectrum in the very special problem of 2D Dirac fermions subject to a random Abelian vector potential. Our result shows that the typical multifractal wave-function spectrum and its termination can be obtained at a generic Anderson localization transition in $d>2$, within the standard field theoretical framework of the nonlinear sigma model, when combined with the FRG.
\end{abstract}

DOI: 10.1103/PhysRevB.80.075101

PACS number(s): 71.30.+h, 72.15.Rn, 05.45.Df, 64.60.al

\section{INTRODUCTION}

Quantum interference induced by multiple elastic impurity scattering can produce very complex spatial fluctuations in electronic wave functions. The statistics of these fluctuations may be used to distinguish different regimes of qualitative wave-function behavior, e.g., localized versus extended. Of particular interest are the wave-function statistics at a delocalization transition, such as the Anderson metalinsulator transition (MIT) (Ref. 1) at the mobility edge in three spatial dimensions, ${ }^{2,3}$ or the integer quantum Hall plateau (IQHP) transition in two dimensions. ${ }^{3-5}$ Here, the spatial structure of the critical wave functions is known not to be characterized by just a single (or a few) independent exponent(s), but by an infinite set thereof ("multifractality"). More precisely, wave-function statistics are encoded through the $\tau(q)$ spectrum or its Legendre transform, the singularity spectrum $f(\alpha) .^{2-7}$

The $\tau(q)$ spectrum is defined via the (generalized) inverse participation ratio (IPR), ${ }^{8}$ given by

$$
P_{q}\left(\varepsilon_{i}\right) \equiv \int_{L^{d}} d^{d} \mathbf{r}\left|\psi_{i}(\mathbf{r})\right|^{2 q}, \quad P_{q} \sim L^{-\tau(q)},
$$

where $d$ is the spatial dimensionality of the system, $L^{d}$ denotes the system volume, and $\left|\psi_{i}(\mathbf{r})\right|^{2}$ is the probability density of a normalized eigenstate wave function $\psi_{i}(\mathbf{r})$ with en$\operatorname{ergy} \varepsilon_{i}$, evaluated at the point $\mathbf{r}$. For eigenenergies $\varepsilon$ lying within a band of extended plane-wave states, $\tau(q)=d(q-1)$, while exponentially localized states yield $\tau(q) \sim 0$ for $L \gg \zeta$, with $\zeta$ the localization length. Multifractal behavior refers to nonlinear $q$ dependence of the $\tau(q)$ spectrum and occurs, e.g., at the mobility edge $\varepsilon=\varepsilon_{c}$ in a disordered threedimensional (3D) system of noninteracting electrons. ${ }^{9}$ The singularity spectrum $f(\alpha)$ is related to the $\tau(q)$ spectrum through the Legendre transformation

$$
f(\alpha)=q \alpha-\tau(q), \quad \frac{d \tau(q)}{d q}=\alpha .
$$

The set of points at which an eigenfunction takes the value $|\psi(\mathbf{r})|^{2} \sim L^{-\alpha}$ is distributed according to the weight $L^{f(\alpha)} ;, 4,10$ in this sense, the singularity spectrum characterizes the interwoven fractal measures of the sample associated with differently scaling components of wave-function intensity. The wave-function statistics have been studied experimentally using thin microwave cavities; ${ }^{11}$ a very broad distribution of the wave-function intensity, indicative of multifractal behavior, was indeed observed. ${ }^{2,6,12}$ In a very recent development, scanning tunneling spectroscopy has been used to map the local density of states of extended and localized wave functions across the IQHP transition. ${ }^{13}$

The multifractal spectrum $[\tau(q)$ or $f(\alpha)]$ at a delocalization critical point is universal and thus serves as a "fingerprint" of the spatial structure of wave functions. Spectra have been computed numerically at myriad delocalization transitions occurring in various spatial dimensions (see, e.g., Refs. 4, 5, and 14-23). In particular, extensive numerical studies of the IQHP transition ${ }^{5,15,17}$ employing different microscopic models have convincingly established the universality of the 
entire $f(\alpha)$ spectrum. Recent work includes that of Refs. 19 and 20, which aim in part at decrypting the critical (conformal field) theory describing the plateau transition.

To compute the entire multifractal spectrum analytically is, however, a very difficult task in generic systems. This is even more so because it is a nonanalytic function of $q$ or $\alpha$. As emphasized in Refs. 3, 24, and 25, this nonanalyticity is related to the fact that the $\tau(q)$ and $f(\alpha)$ spectra are defined for a typical representative wave function, drawn in principle from a system in a single, fixed realization of the static disorder. On the contrary, analytical methods (i.e., those based on field theories) are best suited for calculating quenched averaged quantities. To be precise, we define, following Refs. 24-26, two sets of multifractal statistics in terms of the IPR defined in Eq. (1.1)

$$
\begin{gathered}
\tau(q) \equiv-\frac{d \overline{\left(\ln P_{q}\right)}}{d \ln L}, \\
\tau(q) \equiv-\frac{d \overline{\ln \overline{\left(P_{q}\right)}}}{d \ln L} .
\end{gathered}
$$

In this equation, the overbar $\cdots$ represents an average over realizations of the quenched disorder. The typical $\tau(q)$ spectrum in Eq. (1.1) obtains from the log of the IPR for a representative wave function; since the latter quantity is expected to be self-averaging at the delocalization transition, ${ }^{16,24-26}$ we may introduce an additional, though redundant ensemble average over disorder realizations, as in Eq. (1.3a). We have also defined $\widetilde{\tau}(q)$ in Eq. (1.3b), which obtains from the average of the IPR itself. The averaged IPR can be encoded through the moments of the local density of states (LDOS) operator in an effective low-energy field theory (see Sec. II, below); then, the scaling dimensions of the LDOS moment operators directly determine $\tilde{\tau}(q)$. No such effectively local construction exists for the typical spectrum $\tau(q)$, and in fact "nonlocal" (or more precisely, "multilocal") correlations play an essential role ${ }^{27-30}$ in the "termination" (defined below) of the typical $\tau(q)$, as we show in this paper.

For not too large $|q|$, one expects that

$$
\tau(q)=\widetilde{\tau}(q),
$$

which is the case when the IPR $P_{q}$ represents a selfaveraging quantity [see Sec. III C for a review]. At sufficiently large $|q|$, however, $P_{q}$ becomes broadly distributed $^{2,12,16,24,25,31}$ and the corresponding $\widetilde{\tau}(q)$ spectrum, dominated now by "rare events" induced by the disorder averaging procedure, deviates from $\tau(q) .{ }^{32}$ While $\tilde{\tau}(q)$ is always easier to evaluate analytically, it is $\tau(q)$ that is most easily obtained from a representative wave function in numerics. ${ }^{4,5}$ By comparison, the average $\tilde{\tau}(q)$ and $\tilde{f}(\alpha)$ spectra were computed only recently via numerics at the IQHP (Ref. 17) and Anderson ${ }^{18,22}$ transitions. $^{33}$

In this paper, we calculate the typical multifractal spectrum at the Anderson MIT in the unitary ${ }^{1}$ (broken timereversal) symmetry class of disordered, normal metals in $d$ $>2$. The spectrum $\tilde{\tau}(q)$ associated to the averaged IPR, evaluated at the metal-insulator transition in $d=2+\epsilon$, was obtained long ago $\mathrm{o}^{8,34-36}$ via standard perturbative renormalization group (RG). The form of the typical $\tau(q)$ has been argued before only on heuristic grounds. ${ }^{3,25}$ We compute here the typical spectrum directly using an (analytical) functional renormalization-group (FRG) scheme $^{28-30}$ previously employed in the study of wave-function statistics in a special class of disordered Dirac fermion models in two dimensions (2D). ${ }^{24,26-30,37-42}$

\section{A. Average vs typical spectra and termination}

In the field theory description of Anderson localization [especially the nonlinear sigma model (NL $\sigma \mathrm{M})$ formulation $^{1,43}$ reviewed in Sec. II], the exponent $\tilde{\tau}(q), q$ $\in \mathbb{N}$ of the averaged IPR can be read off from the scaling dimensions $x_{q}^{*}$ and $x_{1}^{*}$ of local composite operators $\mathcal{O}_{q}(\mathbf{r})$ and $\mathcal{O}_{1}(\mathbf{r})$, which represent the $q$ th and first moments of the LDOS, respectively, $8,44,45$

$$
\widetilde{\tau}(q)=d(q-1)+x_{q}^{*}-q x_{1}^{*} .
$$

(See Sec. II for details.) For example, at the Anderson metalinsulator transition in $d=2+\epsilon$ dimensions in the unitary symmetry class, one obtains ${ }^{34-36}$

$$
\begin{gathered}
x_{q}^{*}=-\Xi q(q-1)+\boldsymbol{O}\left[\boldsymbol{\epsilon}^{2} q^{2}(q-1)^{2}\right], \\
x_{1}^{*}=0, \\
\Xi=\sqrt{\boldsymbol{\epsilon} / 2}+\boldsymbol{O}\left(\boldsymbol{\epsilon}^{5 / 2}\right) .
\end{gathered}
$$

We can define a corresponding average singularity spectrum via

$$
\begin{gathered}
\tilde{f}(\alpha) \equiv q \alpha-\tilde{\tau}(q), \quad \frac{d \widetilde{\tau}(q)}{d q}=\alpha, \\
=d-\widetilde{f}_{2}\left(\alpha-\alpha_{0}\right)^{2}+\boldsymbol{O}\left[\sqrt{\boldsymbol{\epsilon}}\left(\alpha-\alpha_{0}\right)^{3}\right],
\end{gathered}
$$

where

$$
\begin{gathered}
\tilde{f}_{2}=\frac{1}{4 \Xi}+\boldsymbol{O}(\boldsymbol{\epsilon}), \\
\alpha_{0}=d+\Xi+\boldsymbol{O}\left(\boldsymbol{\epsilon}^{5 / 2}\right) .
\end{gathered}
$$

The corrections to $[\boldsymbol{O}(\cdots)$ terms in $]$ Eqs. (1.6a), (1.6c), (1.7), (1.8a), and (1.8b) obtain at the fourth loop $\operatorname{order}^{36}$ (or beyond) in the epsilon expansion. By contrast, Eq. (1.6b) is exact and is equivalent to the statement that the average (global) density of states is noncritical at the MIT in the unitary symmetry class. ${ }^{46-48}$ In the present paper, we work only to the lowest nontrivial order in the expansion parameter $\sqrt{\epsilon}$. The consistency of the $\epsilon$ expansion in dealing with high moments of the LDOS operator is demonstrated in Sec. IV. Results similar to Eqs. (1.6a), (1.6b), and (1.6c) were first computed for the time-reversal invariant orthogonal ${ }^{8,49}$ symmetry class. The so-obtained $\widetilde{f}(\alpha)$ spectrum is consistent with largescale numerics. ${ }^{18}$

If one were to reconstruct the probability distribution of the wave-function amplitudes from the average spectra [Eqs. 
(1.5) and (1.7)], a quadratic $\alpha$ dependence of $\tilde{f}(\alpha)$ implies log-normal asymptotics of the distribution function. ${ }^{44,45,50-52}$ The precursor of this broad distribution is already visible at the crossover from the ballistic to diffusive regime, where wave functions start to show (weak) Anderson localization. ${ }^{1}$ In this "prelocalized" regime, renormalization-group studies of an (extended) $\mathrm{NL} \sigma \mathrm{M},{ }^{44,45}$ as well as semiclassical analyses of the supersymmetric (SUSY) NL $\sigma \mathrm{M}$ (Refs. 50-52) predict that the distribution of the wave-function amplitudes starts to deviate from the Gaussian, developing a log-normal tail. ${ }^{6,45}$ As it obtains from the $\widetilde{\tau}(q)$ spectrum associated with the average of the IPR, ${ }^{44,45}$ this tail reflects the influence of rare realizations of the disorder and so-called "anomalously localized states." ${ }^{50-52}$ Even though the tail of the distribution is still small, describing rare events in the mesoscopic regime, it is responsible for anomalous current relaxation, which is slower than expected from the Drude formula. ${ }^{45,50}$

For small $\sqrt{\epsilon}$ (i.e., weak disorder) and $q$ not too large, one might be inclined to expect that the results for $\widetilde{\tau}(q)$ and $\tilde{f}(\alpha)$ in Eqs. (1.5)-(1.8b) should not differ substantially from $\tau(q)$ and $f(\alpha)$, respectively. However, the range of applicability of Eq. (1.7) to the typical $f(\alpha)$ is limited to $\alpha_{-} \leq \alpha \leq \alpha_{+}$, where, to lowest order

$$
\alpha_{ \pm} \equiv(\sqrt{d} \pm \sqrt{\Xi})^{2}+\cdots,
$$

so that $f\left(\alpha_{ \pm}\right)=0$. For $\alpha>\alpha_{+}$and $\alpha<\alpha_{-}$, the average singularity spectrum $\tilde{f}(\alpha)$ becomes negative, which does not make sense if it is interpreted for a typical wave function [see the discussion following Eq. (1.2), above]. These thresholds define the critical values $q_{c}^{ \pm}$of $q$ for the $\tau(q)$ spectrum through

$$
\begin{gathered}
q_{c}^{ \pm} \equiv \frac{d \widetilde{f}\left(\alpha_{\overline{+}}\right)}{d \alpha}= \pm q_{c}+\cdots, \\
q_{c}=\sqrt{\frac{d}{\Xi}} \sim\left(\frac{8}{\epsilon}\right)^{1 / 4}+\boldsymbol{O}\left(\epsilon^{1 / 4}\right) .
\end{gathered}
$$

[The IPR $P_{q}$, as well as the typical and average multifractal spectra $\tau(q)$ and $\widetilde{\tau}(q)$ can be extended to negative $q$ using the notion of box probabilities ${ }^{4,5}$ - see Ref. 53 for details.]

For $q>q_{c}^{+}, q<q_{c}^{-}$, the typical spectrum $\tau(q)$ deviates completely from the average $\widetilde{\tau}(q)$, given by Eqs. (1.5)-(1.6c) to lowest order in the epsilon expansion. Indeed, it can be rigorously proved ${ }^{4}$ that the $\tau(q)$ spectrum (as defined for a typical wave function) must be a monotonically increasing function of $q$; by comparison, the average spectrum $\widetilde{\tau}(q)$ in Eqs. (1.5)-(1.6c) is monotonically decreasing for $q>(d$ $+\Xi) / 2 \Xi$. For $q>q_{c}^{+}, q<q_{c}^{-}$, the rare maxima (minima) of the wave-function amplitude dominate the IPR [Eq. (1.1)], as computed for a representative wave function in a fixed disorder realization. In this regime, the associated $\tau(q)$ is linear in $q$. By contrast, Eq. (1.4) holds for $q_{c}^{-}<q<q_{c}^{+}$. In Fig. 1, we plot the average spectrum $\widetilde{\tau}(q)$ as given by Eqs. (1.5)-(1.6c), as well as our final result for the typical spectrum $\tau(q)$, which we obtain in Sec. III of this paper [Eq. (3.25), below].

We say that the multifractal behavior of the typical $\tau(q)$ spectrum "terminates" at $q=q_{c}^{ \pm}$. This result in turn implies
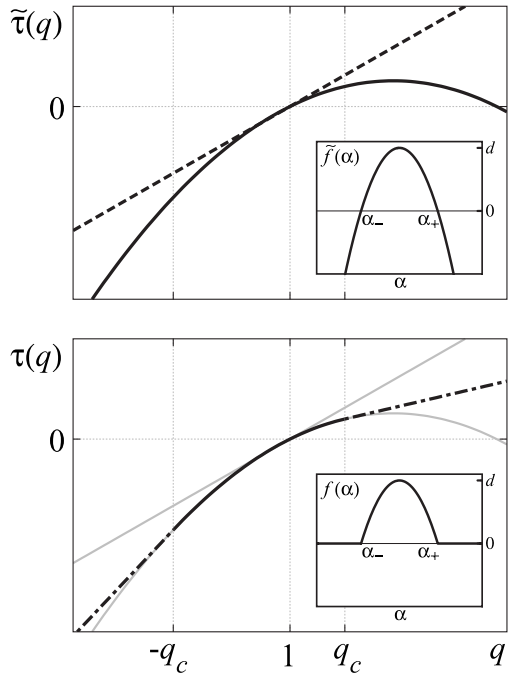

FIG. 1. Sketch of the multifractal spectra at the unitary class Anderson MIT. In the top panel, the heavy solid line corresponds to the average spectrum $\tilde{\tau}(q)$, defined by Eq. (1.3b) in the text, as obtained at the lowest nontrivial order in the $\epsilon$ expansion [Eqs. (1.5)-(1.6c)] (Refs. 34-36). For comparison, the heavy dashed line in the same plot is the linear spectrum for a plane-wave state, $\tilde{\tau}(q)=\tau(q)=d(q-1)$. In the bottom panel, the solid and dot-dashed heavy line segments represent the typical spectrum $\tau(q)$, defined by Eq. (1.3a), as obtained in this paper via the functional renormalization group [see Eq. (3.25)]. For $q_{c}^{-} \leq q \leq q_{c}^{+}$(solid segment of the curve in the bottom panel), the typical and average spectra coincide. By contrast, the typical spectrum is linear for $q<q_{c}^{-}, q>q_{c}^{+}$(beyond "termination"), as depicted by the dot-dashed curve segments in the bottom panel. The two curves in the top panel are rendered as faint gray lines in the bottom, for comparison. Inset in the top (bottom) panel depicts the average (typical) singularity spectrum at the unitary class MIT corresponding to the as-sketched $\widetilde{\tau}(q)[\tau(q)]$.

that the singularity spectrum $f(\alpha)$ must also suffer "termination," i.e., vanish outside of the range bounded by $\alpha_{ \pm}$. The paramount distinction between typical vs average spectra is therefore summarized as follows: the termination of $\tau(q)$ and $f(\alpha)$ reflects the dominance of rare amplitude extrema occurring in a representative wave function computed for a particular configuration of the disorder, whereas the deviation of $\widetilde{\tau}(q)$ and $\widetilde{f}(\alpha)$ from the former reflects the influence of rare disorder realizations that enter into the averaged IPR, $\overline{P_{q}}$ [Eq. (1.3b)].

\section{B. Operator product expansion and the functional renormalization group}

The dimension $x_{q}^{*}(q \in\{1,2, \ldots\})$ in Eqs. (1.5) and (1.6a) describes the scaling of the disorder-averaged $q$ th LDOS moment at criticality, represented by the operator $\mathcal{O}_{q}(\mathbf{r})$. In order to extract the evolution of the typical value of an LDOS moment, we require a scaling equation for its entire probability distribution: a functional RG approach will turn out to be necessary. We will demonstrate that the scaling of the typical LDOS moments determines the $\tau(q)$ spectrum.

A key technical difference distinguishing the calculation of the typical $\tau(q)$ spectrum from that of the average $\widetilde{\tau}(q)$ 
spectrum is that different LDOS moments couple to each other along the FRG flow. This coupling among the moments is encoded in the operator product expansion (OPE) of the scaling operators at the delocalization critical point

$$
\mathcal{O}_{q}(\mathbf{r}) \mathcal{O}_{q^{\prime}}\left(\mathbf{r}^{\prime}\right)=\frac{C_{q, q^{\prime}}^{q+q^{\prime}}}{\left|\mathbf{r}-\mathbf{r}^{\prime}\right| x_{q^{*}+x_{q^{\prime}}^{*} x_{q+q^{\prime}}^{*}}^{*}} \mathcal{O}_{q+q^{\prime}}\left(\frac{\mathbf{r}+\mathbf{r}^{\prime}}{2}\right)+\cdots
$$

Whenever the OPE coefficient $C_{q, q^{\prime}}^{q+q^{\prime}} \neq 0$, lower moments generate higher ones upon the RG transformation. The $\left\{x_{q}^{*}\right\}$ satisfy the convexity relation ${ }^{54}$

$$
x_{q+q^{\prime}}^{*}<x_{q}^{*}+x_{q^{\prime}}^{*}<0
$$

for $q, q^{\prime}>1$. Since the $\left\{x_{q}^{*}\right\}$ are negative here, Eq. (1.12) indicates that higher moments are much more relevant and hence we are forced to retain all mutually coupled moments in the theory without being able to resort to truncation. The FRG allows us to organize and track the entire infinite tower of LDOS moment operators. The nonzero OPE coefficient $C_{q, q^{\prime}}^{q+q^{\prime}}$ leads to a nonlinearity within the FRG; the unbounded broadening suggested by the $q$ dependence of $x_{q}^{*}$ (reflecting the ever more relevant nature of the corresponding operators, with increasing $q$ ) is balanced by this nonlinearity. For smallenough values of $q$, the nonlinearity will entirely offset the unbounded broadening and render it inconsequential, whereas for sufficiently large values of $q$, this will result in the termination of the typical $\tau(q)$ spectrum.

The mechanism described above is known to be responsible for the termination of the multifractal spectrum in a special (so-called "chiral") (Ref. 55) symmetry class of 2D models, possessing quenched disorder. Carpentier and Le Doussal $^{28,29}$ pioneered the use of the FRG technique in their study of the random-phase $X Y$ (gauge glass) model. This method was later applied ${ }^{29}$ to the problem ${ }^{37}$ of a $2 \mathrm{D}$ massless Dirac fermion, subject to a static, random Abelian vector potential. The FRG provided direct confirmation of the multifractal termination for this problem, a result previously conjectured $^{24,38}$ for the vector potential model. Later, Mudry et $a l .{ }^{30}$ extended the FRG to a more general 2D disordered Dirac model belonging to the symmetry class BDI (chiral orthogonal symmetry class). (We have adopted the nomenclature for quantum disorder classes employed in Ref. 55.) In these works, the FRG equation constructed from the set of operator scaling dimensions $x_{q}^{*}$ and OPE coefficients $C_{q, q^{\prime}}^{q+q^{\prime}}$ [Eq. (1.11)] takes the form of the so-called KolmogorovPetrovsky-Piscounov (KPP) equation, ${ }^{56}$ which describes nonlinear diffusion in one dimension. It is the nontrivial behavior of the long-time asymptotics of the solution to the KPP equation that is responsible for the termination. We will show that the same equation arises in the general context of the typical $\tau(q)$ spectrum in the unitary symmetry class at the Anderson MIT critical point in $d=2+\epsilon$ (with obvious extensions to additional symmetry classes).

\section{Outline}

Using the framework of the fermionic replica (compact) NL $\sigma \mathrm{M}$ approach, ${ }^{1,43,57}$ we compute the OPE coefficient $C_{q, q^{\prime}}^{q+q^{\prime}}$ at the critical point in $d=2+\epsilon$ for the unitary class. Combining this result with the scaling dimensions given by Eqs. (1.6a), (1.6b), and (1.6c), we formulate the functional renormalization group for the tower of LDOS moment operators that enters into the computation of the typical $\tau(q)$ spectrum. Then we use the FRG to demonstrate that the same mechanism active in the $2 \mathrm{D}$ Dirac models, ${ }^{28-30}$ discussed above, leads to the termination of the multifractal spectrum at the MIT. We obtain the $\tau(q)$ spectrum for a typical wave function, which agrees with previous heuristic arguments. $^{16,25}$

The rest of the paper is organized as follows. In Sec. II, we review the connection between the IPR and the local density of states and we introduce a generating function that will be used to determine the typical $\tau(q)$ spectrum. We then establish conventions for the fermionic replica NL $\sigma \mathrm{M}$ and identify the composite operators that represent moments of the local density of states in the low-energy field theory. In Sec. III, we use the OPE of the LDOS moment operators at the MIT as input into the FRG, which then allows us to compute the scaling behavior of the generating function introduced in Sec. II. We thereby obtain the typical $\tau(q)$ spectrum. We discuss our results and draw conclusions in Sec. IV.

The derivation of the OPE of the operators $\left\{\mathcal{O}_{q}(\mathbf{r})\right\}$ representing the LDOS moments, which constitutes the technical field-theoretic content of this work, has been relegated to Sec. V. In this section, we rederive the anomalous scaling dimensions of the LDOS moment operators and we compute the required OPE coefficient between properly normalized versions of these. The results obtained are invoked as needed in the earlier Sec. III, so the reader that is less interested in calculational details may skip Sec. V entirely.

\section{DEFINITIONS AND MODEI}

\section{A. Extracting multifractality from the LDOS-typical spectra}

Consider the LDOS, defined as

$$
\nu(\varepsilon, \mathbf{r})=\frac{-1}{\pi} \operatorname{Im} G_{R}(\varepsilon ; \mathbf{r}, \mathbf{r})=\sum_{i} \delta\left(\varepsilon-\varepsilon_{i}\right)\left|\psi_{i}(\mathbf{r})\right|^{2},
$$

where the retarded Green's function is given by

$$
G_{R}\left(\varepsilon ; \mathbf{r}, \mathbf{r}^{\prime}\right)=\sum_{i} \frac{\psi_{i}(\mathbf{r}) \psi_{i}^{*}\left(\mathbf{r}^{\prime}\right)}{\varepsilon-\varepsilon_{i}+i \eta},
$$

with $\eta \rightarrow 0^{+}$. On the metallic side of the delocalization transition, we cannot relate $P_{q}(\varepsilon)$, defined in terms of a single wave function by Eq. (1.1), directly to the $\operatorname{LDOS}^{8}$ In order to use the field theory approach, we require that the LDOS constitute a smooth, well-defined function of energy in a closed, finite-size system; this necessitates the retention of the finite-energy level broadening $\eta \gtrsim \Delta$, where $\Delta$ is the global level spacing. (Although a formal device in this context, the broadening may be attributed to, e.g., inelastic relaxation 
processes neglected in the noninteracting, single-particle approach.)

We define ${ }^{8}$

$$
\frac{1}{L^{d(q-1)} p^{(q)}(\varepsilon)} \equiv \frac{\int d^{d} \mathbf{r} \nu^{q}(\varepsilon, \mathbf{r})}{\left[\int d^{d} \mathbf{r} \nu(\varepsilon, \mathbf{r})\right]^{q}} .
$$

The quantity $p^{(q)}(\varepsilon)$ denotes the participation ratio, which receives contributions from states with energies residing in a window of width $\eta$ about $\varepsilon$. On the metallic side of the transition, the right-hand side (RHS) of Eq. (2.3) should scale identically as Eq. (1.1). ${ }^{8}$

It will prove useful to introduce the moment generating function for the $q$ th power of the LDOS

$$
F_{q}(\xi ; L) \equiv\left\langle\exp \left[-\xi \int d^{d} \mathbf{r} \nu^{q}(\varepsilon, \mathbf{r})\right]\right\rangle,
$$

where the angle brackets $\langle\cdots\rangle$ denote a suitable ensemble average over realizations of the quenched disorder; $L$ is the linear system size. Using the identity

$$
\ln \phi=\int_{0}^{\infty} \frac{d \xi}{\xi}\left(e^{-\xi}-e^{-\phi \xi}\right)
$$

and replacing $P_{q}$ with the RHS of Eq. (2.3) in Eq. (1.3a), the typical multifractal spectrum exponent $\tau(q)$ may be written as

$$
\tau(q)=\frac{d}{d \ln L} \int_{0}^{\infty} \frac{d \xi}{\xi}\left[F_{q}(\xi ; L)-q F_{1}(\xi ; L)\right] .
$$

Our goal is to compute the scaling behavior of the momentgenerating function $F_{q}(\xi ; L)$ and thereby obtain the typical $\tau(q)$ spectrum via Eq. (2.6). In closing this section, we note that the evaluation of Eq. (2.6) using the lowest-order cumulant expansion for $F_{q}(\xi ; L)$ recovers the average $\widetilde{\tau}(q)$ spectrum, Eq. (1.5); we will discuss this point in detail in Sec. III. [See Eq. (3.3) and the text that follows it.]

\section{B. NL $\sigma$ M formulation}

We examine in this paper the properties of the multifractal spectrum at the Anderson MIT in the unitary symmetry class. The critical point itself is accessed via the standard perturbative $\epsilon$ expansion in $d=2+\epsilon$ dimensions, with $0<\epsilon \ll 1$. Our low-energy, effective-field theory starting point is the compact replica NL $\sigma \mathrm{M}, 1,43,57$ defined by the functional integral

$$
Z \equiv \int \mathcal{D}[\hat{Q}] e^{-S}
$$

where

$$
S[\hat{Q}] \equiv \frac{1}{2 t} \int d^{d} \mathbf{r} \operatorname{Tr}(\boldsymbol{\nabla} \hat{Q} \cdot \boldsymbol{\nabla} \hat{Q})-h \int d^{d} \mathbf{r} \operatorname{Tr}\left(\hat{\Lambda}_{z} \hat{Q}\right) .
$$

In this equation, the "temperature" $t$ is inversely proportional to the dimensionless dc conductance of the disordered metal, while the "external field" $h$ serves as an infrared regulator, coupling to the LDOS operator, as defined below. The symbol $\hat{Q}$ denotes a $2 n \times 2 n$ Hermitian matrix field satisfying

$$
\hat{Q}^{2}(\mathbf{r})=\hat{\mathbb{I}}_{2 n}, \quad \operatorname{Tr} \hat{Q}(\mathbf{r})=0 .
$$

The constant matrix

$$
\hat{\Lambda}_{z}=\operatorname{diag}\left(\hat{\mathbb{I}}_{n},-\hat{\mathbb{I}}_{n}\right)
$$

sets the (trivial) saddle point for the action defined by Eq. (2.7). The identity in the space of $2 n \times 2 n$ and $n \times n$ square matrices is denoted by $\hat{\mathbb{I}}_{2 n}$ and $\hat{\mathbb{I}}_{n}$ in Eqs. (2.8) and (2.9), respectively. In these equations, $n$ is proportional to the number of replicas, with $n \rightarrow 0$ at the end of the calculation. ${ }^{1,43}$ The target space of the NL $\sigma \mathrm{M}$ is the compact coset $G(2 n) / G(n) \times G(n)$, where $G=O, U, S p$ for the orthogonal, unitary, and symplectic symmetry classes, respectively. In the following, we focus upon the unitary universality class, $G=U$. At the same time, we stress that the functional renormalization-group procedure presented in Sec. III is completely general and can be applied to the termination of the typical multifractal spectrum at any critical delocalization fixed point, given an appropriate tower of LDOS operators, their scaling dimensions, and operator product expansion coefficients. The field theory in Eqs. (2.7) and (2.8) can be derived $^{57}$ from a microscopic Grassmann path integral describing a system of noninteracting fermions, lacking timereversal invariance, averaged over configurations of a Gaussian, white noise-correlated random potential.

We employ " $\sigma-\pi$ " coordinates ${ }^{47}$ on the target manifold

$$
\hat{Q}=\left[\begin{array}{cc}
\left(\hat{\mathbb{I}}_{n}-\hat{W} \hat{W}^{\dagger}\right)^{1 / 2} & \hat{W} \\
\hat{W}^{\dagger} & -\left(\hat{\mathbb{I}}_{n}-\hat{W}^{\dagger} \hat{W}\right)^{1 / 2}
\end{array}\right] .
$$

For the unitary class, $\hat{W}(\mathbf{r}) \rightarrow W_{\beta}^{\alpha}(\mathbf{r})$ is an unconstrained, complex-valued matrix, with $\alpha, \beta \in\{1, \ldots, n\}$.

Noninteracting electrons residing in $d>2$ spatial dimensions and subject to quenched disorder possess a diffusive metallic phase, defined as the presence of extended wave functions at the Fermi energy, provided that the disorder is sufficiently weak. The disorder strength is quantified by the "bare" conductance at the scale of the mean-free path, proportional to $1 / t$ in the effective-field theory [Eq. (2.7)]. In direct analogy with the $O(3) / O(2)$ NL $\sigma \mathrm{M}$ description of classical magnetic ordering, ${ }^{58-60}$ the "low-temperature" (weak disorder) regime $0 \leq t<t^{*}$ of the model in Eq. (2.7) exhibits spontaneous continuous symmetry breaking, so that the " $\sigma$ " fields $\left(\hat{\mathbb{I}}_{n}-\hat{W} \hat{W}^{\dagger}\right)^{1 / 2}$ and $\left(\hat{\mathbb{I}}_{n}-\hat{W}^{\dagger} \hat{W}\right)^{1 / 2}$, which form the diagonal elements of the $\hat{Q}$ matrix in the parameterization of Eq. (2.10), acquire nonzero expectation values throughout the diffusive metallic phase. By contrast, the off-diagonal " $\pi$ " fields $\hat{W}$ and $\hat{W}^{\dagger}$ represent small spatial fluctuations with vanishing mean in this regime. Here, $t=t^{*}>0$ locates the MIT in $d=2+\epsilon$.

An unusual aspect of the theory of the MIT transcribed in Eq. (2.7) is the fact that this spontaneous symmetry breaking occurs also at the delocalization transition itself $\left(t=t^{*}\right)$ and survives even into the insulating ("high-temperature") phase 
$\left(t>t^{*}\right) \cdot{ }^{46,47}$ In the effective NL $\sigma \mathrm{M}$ field theory, the trace of the matrix $\hat{\Lambda}_{z} \hat{Q}(\mathbf{r})$ represents the LDOS $\nu(\varepsilon, \mathbf{r})$ [Eq. (2.1)] for the disordered electron system ${ }^{8}$

$$
\begin{aligned}
\nu(\varepsilon, \mathbf{r}) \sim & \operatorname{Tr}\left[\hat{\Lambda}_{z} \hat{Q}(\mathbf{r})\right] \\
= & \operatorname{Tr}\left\{\left[\hat{\mathbb{I}}_{n}-\hat{W} \hat{W}^{\dagger}(\mathbf{r})\right]^{1 / 2}\right. \\
& \left.+\left[\hat{\mathbb{I}}_{n}-\hat{W}^{\dagger} \hat{W}(\mathbf{r})\right]^{1 / 2}\right\} .
\end{aligned}
$$

This is the same operator that appears in the action Eq. (2.7), where it couples to the external field parameter $h$. While the character of the typical wave function changes from extended to localized upon traversing the mobility edge, as encoded by, e.g., the typical multifractal exponent $\tau(q)$ for $q \geq 2$ [Eq. (2.6)], the average density of states does not exhibit critical behavior across the transition. ${ }^{46}$ The LDOS operator on the RHS of Eq. (2.11) retains a nonzero expectation value so long as the average density of states is nonvanishing; consequently, the $\hat{Q}$ matrix cannot be interpreted as an order parameter for the MIT. Technically, this result (an exception to Goldstone's theorem) (Ref. 47) obtains from the $\mathrm{NL} \sigma \mathrm{M}$ only after the replica limit $n \rightarrow 0$ is taken.

For any nonzero, integral number of replicas $n$ $\in\{1,2, \ldots\}$, the model in Eq. (2.7) also possesses a (different) second-order transition at $t=t_{n}^{*}>0$, separating a lowtemperature "ferromagnetic" phase $\left(t<t_{n}^{*}\right)$ from the hightemperature "paramagnet" $\left(t>t_{n}^{*}\right)$. In contrast to the replica limit $n \rightarrow 0$ appropriate to the description of electronic wavefunction (de)localization, the NL $\sigma \mathrm{M}$ with $n \geq 1$ is characterized by a restoration of the symmetry at the critical point between the $\sigma$ (diagonal) and $\pi$ (off-diagonal) components of the $\hat{Q}$ matrix within the parameterization given by Eq. (2.10). This is the conventional behavior expected for a classical statistical-mechanics model describing spontaneous continuous symmetry breaking in the vicinity of the critical point.

Let us assume that we are interested only in properties of the NL $\sigma \mathrm{M}$ given by Eq. (2.7) at the critical point, $t=t_{n}^{*}$. Because the symmetry is restored at the transition, for nonzero $n$, we are permitted to make the following $U(2 n)$ "rotation" from $\hat{\Lambda}_{z}$ to $\hat{\Lambda}_{x}$ in Eq. (2.11):

$$
\nu(\varepsilon, \mathbf{r}) \sim \operatorname{Tr}\left[\hat{\Lambda}_{z} \hat{Q}(\mathbf{r})\right] \rightarrow \operatorname{Tr}\left[\hat{\Lambda}_{x} \hat{Q}(\mathbf{r})\right]=\operatorname{Tr}\left[\hat{W}(\mathbf{r})+\hat{W}^{\dagger}(\mathbf{r})\right],
$$

where $\hat{\Lambda}_{x}$ denotes the block Pauli matrix generalizing Eq. (2.9) in the standard basis.

In the technical field-theoretic portion of this paper, Sec. $\mathrm{V}$, we employ the NL $\sigma \mathrm{M}$ defined by Eqs. (2.7)-(2.10) to extract the properties of the LDOS operator and its moments. Our strategy is to work, as usual, at fixed, integral $n \geq 1$ throughout the intermediate stages of our computations. At the critical point in $d=2+\epsilon$, we are then free to employ the LDOS representation given by the RHS of Eq. (2.12). Only at the end of our work will we perform the required analytic continuation $n \rightarrow 0$ (which smoothly deforms $t_{n}^{*} \rightarrow t^{*}$ ), so as to obtain (perturbative) results appropriate to the MIT.

\section{LDOS moments as composite eigenoperators}

Higher integral moments of the LDOS can be similarly represented by local composite operators in the NL $\sigma \mathrm{M}$. The $\mathrm{RG}$ transformation does not preserve the form of an operator

$$
\nu^{p}=\left[\operatorname{Tr}\left(\hat{W}+\hat{W}^{\dagger}\right)\right]^{p},
$$

obtained by taking a power of Eq. (2.12). Nevertheless, such a structure can be decomposed into invariant eigenoperators, each of which possessing an independent scaling dimension.

This idea is most easily understood via analogy to the simpler $O(3) / O(2)$ model, ${ }^{58-60}$ to which the field theory defined by Eqs. (2.7)-(2.10) reduces for the case of $n=1$ [since $U(2) / U(1) \times U(1) \sim S U(2) / U(1) \sim O(3) / O(2)]$. In this NL $\sigma \mathrm{M}$, the target manifold is simply the two-sphere, parameterized by the unconstrained transverse coordinates $\pi_{ \pm}$ $\equiv \pi_{x} \pm i \pi_{y}$, with $z$ component $\sigma=\sqrt{1-\pi_{+} \pi_{-}}$. A complete basis of local eigenoperators with no derivatives is the set of ordinary spherical harmonics $\left\{Y_{l, m}\left(\pi_{+}, \pi_{-}, \sigma\right)\right\}$. All operators belonging to a given irreducible representation of the symmetry group possess the same renormalization; therefore, any linear combination of spherical harmonics sharing a common $l$ value constitutes an eigenoperator. The field coordinates $\pi_{ \pm}$are themselves eigenoperators belonging to $l=1$, as is the combination

$$
\nu \equiv \pi_{+}+\pi_{-} \propto Y_{1,-1}-Y_{1,1} .
$$

For an arbitrary integer moment of $\nu$, one can use angularmomentum addition to establish the decomposition

$$
\left(\pi_{+}+\pi_{-}\right)^{l}=\sum_{j=0}^{l} \mathcal{O}_{j}^{(l)},
$$

where the eigenoperators $\mathcal{O}_{j}^{(l)}$ are defined via

$$
\mathcal{O}_{j}^{(l)}=\sum_{m=-j}^{j} \kappa_{j, m}^{(l)} Y_{j, m}\left(\pi_{+}, \pi_{-}, \sigma\right),
$$

with certain coefficients $\kappa_{j, m}^{(l)}$. For the highest total angularmomentum block $j=l$ in Eq. (2.15), one has

$$
\mathcal{O}_{l}^{(l)}=\left(\pi_{-}^{l}+\cdots+\pi_{+}^{l}\right),
$$

since the "highest and lowest weight states" $\pi_{+}^{l}$ and $\pi_{-}^{l}$ are eigenoperators proportional to $Y_{l, l}$ and $Y_{l,-l}$, respectively.

The coefficients $\left\{\kappa_{j, m}^{(l)}\right\}$ on the RHS of Eq. (2.16) are determined entirely by group theory (i.e., are composed of sums of products of appropriate Clebsch-Gordan coefficients), ${ }^{61}$ up to an overall $m$-independent normalization for all operators belonging to a given total angularmomentum block $j$. This normalization can be established via the convention

$$
Y_{l,-l} \equiv \lambda_{l} \pi_{-}^{l} .
$$

In a similar fashion, the operator in Eq. (2.13) should be decomposed into a sum of terms belonging to different irreducible representations of the group $U(2 n)$. Each such term can be further decomposed into a linear combination of basis operators with appropriate "magnetic" quantum numbers determined by the transformation properties under the subgroup $U(n) \times U(n)$. 
It is useful to push this analogy a little further. In order to extract the typical $\tau(q)$ spectrum in the unitary class model, we need the scaling dimension of the most relevant eigenoperator (in the RG sense) contributing to each of the $p$ th LDOS moments in Eq. (2.13), $p \in\{1,2, \ldots\}$, as well as the OPE between pairs of such most relevant eigenoperators. The most relevant eigenoperator contributing to the decomposition of Eq. (2.13), for a given fixed $p$, is analogous to the highest (total) angular-momentum operator $\mathcal{O}_{l}^{(l)}$ contributing to the $l$ th moment of $\left(\pi_{+}+\pi_{-}\right)$in Eq. $(2.15),{ }^{62}$ with $l=p$. [Precise definitions of the eigenoperators that we employ in the $U(2 n) / U(n) \times U(n) \mathrm{NL} \sigma \mathrm{M}$ are given by Eqs. (2.23) and (2.24), below.] In the $O(3) / O(2)$ model, we can effectively trade the operator $\mathcal{O}_{l}^{(l)}$, which for large $l$ is a complicated sum of many terms according to Eq. (2.16), for its lone "lowest-weight state" component $Y_{l,-l}$ [Eqs. (2.17) and (2.18)]. Obviously, both operators share the same scaling dimension. Moreover, the structure of the OPE between $\mathcal{O}_{l}^{(l)}$ and $\mathcal{O}_{l^{\prime}}^{\left(l^{\prime}\right)}$ follows from that of the product between their lowest weight state constituents. Consider the following OPE at zero coupling $(t=0)$,

$$
\begin{gathered}
\lambda_{l} \lambda_{l^{\prime}} \mathcal{O}_{l}^{(l)} \mathcal{O}_{l^{\prime}}^{\left(l^{\prime}\right)}=c_{l, l^{\prime}}^{l+l^{\prime}} \lambda_{l+l^{\prime}} \mathcal{O}_{l+l^{\prime}}^{\left(l+l^{\prime}\right)}, \\
\left(Y_{l,-l}+\cdots\right)\left(Y_{l^{\prime},-l^{\prime}}+\cdots\right)=c_{l, l^{\prime}}^{l+l^{\prime}}\left(Y_{l+l^{\prime},-l-l^{\prime}}+\cdots\right),
\end{gathered}
$$

where we have defined the OPE coefficient

$$
c_{l, l^{\prime}}^{l+l^{\prime}} \equiv \frac{\lambda_{l} \lambda_{l^{\prime}}}{\lambda_{l+l^{\prime}}} .
$$

The crucial point is that the relative weight of each term appearing in the expansion for the eigenoperator $\mathcal{O}_{l}^{(l)}$ [Eq. (2.16)] is entirely fixed by group theory; only the overall $l$-dependent normalization is arbitrary. The required OPE coefficient in Eq. (2.20) is then determined by just this normalization for the lowest weight state operators, Eq. (2.18). Of course, this argument neglects loop corrections, which may modify the value of the OPE coefficient given by Eq. (2.20), computable systematically within the $\epsilon$ expansion. This, however, cannot alter the structure of Eq. (2.19).

With the above in mind, we consider the component

$$
[\operatorname{Tr} \hat{W}]^{p}
$$

of the LDOS moment in Eq. (2.13). As opposed to the sphere model discussed above, this pure $\hat{W}$ power does not represent an eigenoperator for $n>1$. However, a useful subset ${ }^{63}$ of the RG eigenoperators can be built out of $p$-fold products of $\pi$ $\left(W_{\beta}^{\alpha}\right)$ field matrix elements

$$
\mathcal{O}_{p\left(\beta_{1} \beta_{2} \ldots \beta_{p}\right) y}^{\alpha_{1} \alpha_{2} \ldots \alpha_{p}}(\mathbf{r}) \equiv \frac{1}{p !} W_{\left(\beta_{1}\right.}^{\alpha_{1}} W_{\beta_{2}}^{\alpha_{2}} \ldots W_{\left.\beta_{p}\right)}^{\alpha_{1}},
$$

where $(\cdots)_{y}$ means a suitable symmetrization prescribed by a Young tableau $\mathcal{Y}$. For fixed $p$, the most relevant operator (in the sense of the RG, at the MIT in $d=2+\epsilon$ ) is given by the totally antisymmetric Young tableau ${ }^{8,34}$

$$
\mathcal{O}_{p\left[\beta_{1} \beta_{2} \ldots \beta_{p}\right]}^{\alpha_{1} \alpha_{2} \ldots \alpha_{p}}(\mathbf{r}) \equiv\left(\frac{1}{p !}\right)^{2} \sum_{\mathbf{P}} \operatorname{sgn}(\mathbf{P})\left[W_{\beta_{\mathbf{P}(1)}}^{\alpha_{1}} \ldots W_{\beta_{\mathbf{P}(p)}}^{\alpha_{p}}\right],
$$

with $\mathbf{P}$ a permutation of $p$ symbols; $\operatorname{sgn}(\mathbf{P})$ denotes the sign of the permutation. Because of the antisymmetrization requirement, each distinct operator defined through Eq. (2.23) is identified by any permutation of a complete set of indices $\left\{\alpha_{i}\right\}$ satisfying $\alpha_{1} \neq \alpha_{2} \neq \cdots \neq \alpha_{p}$ and similarly for the $\left\{\beta_{i}\right\}$. Indices range from 1 to $n$, so that many different operators can be associated to each integral moment of the LDOS, at least for sufficiently large $n$.

Physically, we would like establish a one-to-one correspondence between the $p$ th $\operatorname{LDOS}$ moment $[\nu(\varepsilon, \mathbf{r})]^{p}$ in the disordered electron system and a single, unique operator $\mathcal{O}_{p}$ in the NL $\sigma \mathrm{M}$ field theory that represents its most relevant component. This can be accomplished by tracing over pairs of indices in Eq. (2.23) in the following fashion:

$$
\mathcal{O}_{p}(\mathbf{r}) \equiv \sum_{\alpha_{1}=1}^{n} \ldots \sum_{\alpha_{p}=1}^{n} \mathcal{O}_{p\left[\alpha_{1} \alpha_{2} \ldots \alpha_{p}\right]}^{\alpha_{1} \alpha_{2} \ldots \alpha_{p}}(\mathbf{r}) .
$$

With this definition, the eigenoperators

$$
\mathcal{O}_{2}=\frac{[\operatorname{Tr}(\hat{W})]^{2}-\operatorname{Tr}\left(\hat{W}^{2}\right)}{(2 !)^{2}},
$$

$$
\mathcal{O}_{3}=\frac{[\operatorname{Tr}(\hat{W})]^{3}-3 \operatorname{Tr}\left(\hat{W}^{2}\right) \operatorname{Tr}(\hat{W})+2 \operatorname{Tr}\left(\hat{W}^{3}\right)}{(3 !)^{2}},
$$

etc., are easily recognized as natural deformations of the LDOS moments obtained by taking powers of Eq. (2.21). ${ }^{63}$ Moreover, we will establish in Sec. V that the set $\left\{\mathcal{O}_{p}\right\}$ closes under the OPE up to less relevant operators generated on the right-hand side of Eq. (1.11), which we may ignore. This is a sufficient condition to apply the functional renormalizationgroup method. In summary, the operators defined by Eqs. (2.23) and (2.24) constitute the most relevant component(s) of the $p$ th moment of the $\operatorname{LDOS}^{8,34,63}$ at the MIT and hence dominate its scaling behavior there.

\section{Augmented NLoM}

At the metal-insulator critical point, the scaling of the average IPR $P_{q}$ [i.e., the multifractal exponent $\widetilde{\tau}(q)$, Eq. $(1.3 \mathrm{~b})]$ can be extracted solely from the scaling dimensions $x_{p}^{*}$ of the local composite operators $\mathcal{O}_{p\left[\beta_{1} \beta_{2} \cdots \beta_{p}\right]}^{\alpha_{1} \alpha_{2} \ldots \alpha_{p}}(\mathbf{r})$ or $\mathcal{O}_{p}(\mathbf{r})$, with $p \in\{1, q\}$ - this is the content of Eq. (1.5) in Sec. I. By contrast, the probability distribution functions of the IPR and LDOS [reflected by the typical multifractal exponent $\tau(q)$, Eq. (1.3a)] are described by the complicated generating function $F_{q}(\xi ; L)$, introduced in Eq. (2.4). In the low-energy theory, $F_{1}(\xi ; L)$ can be represented by the NL $\sigma \mathrm{M}$ in Eq. (2.7) with a bare nonzero external field parameter $h_{0}$ given by

$$
h_{0}=-\xi \text {. }
$$

Performing a renormalization-group transformation upon the $\mathrm{NL} \sigma \mathrm{M}$ with $h_{0} \neq 0$ generically produces higher powers of 
the LDOS operator as new perturbations to the action $S$, so that terms of the form

$$
\delta S=-y_{m} \int d^{d} \mathbf{r}\left\{\operatorname{Tr}\left[\hat{\Lambda}_{z} \hat{Q}(\mathbf{r})\right]\right\}^{m},
$$

for example, will be generated. Here, $y_{m}$ is a coupling constant. The structure in Eq. (2.26) is not invariant under the $\mathrm{RG}$; with further iterations, it will (a) mix with other terms sharing the same "engineering" dimension and (b) fuse with other terms and with itself to produce new perturbations. Among the flood of structures that arise, we will focus only upon the most relevant terms that determine the leading scaling behavior for the generating function $F_{1}(\xi ; L)$ of the LDOS and its moments. Anticipating the generation of higher moments upon renormalization, we should augment the action in Eq. (2.7) (with $h_{0}=0$ ) by a term of the form

$$
\delta S \equiv-\sum_{p=1}^{\infty} y_{p} \int d^{d} \mathbf{r} \mathcal{O}_{p}(\mathbf{r})
$$

where the "traced" moment operators $\mathcal{O}_{p}$ were defined above by Eq. (2.24).

At tree level, the operators defined by Eqs. (2.23) and (2.24) are dimensionless, so that the corresponding coupling constants $\left\{y_{p}\right\}$ are strongly relevant perturbations to the $\mathrm{NL} \sigma \mathrm{M}$ action. As discussed in Sec. I B, they prove even more relevant at the nontrivial fixed point (perturbatively accessible Anderson MIT). Moreover, the higher moments are more relevant compared to the lower ones [Eq. (1.12)]. The FRG approach tracks the scaling behavior of this entire tower of operators and uses this data to make nontrivial predictions about observable statistics, such as the typical LDOS. Within the FRG framework, only two pieces of information are needed: first, the scaling dimensions of the operators in Eqs. (2.23) and (2.24) and second, the coefficient $C_{q, q^{\prime}}^{q+q^{\prime}}$ for the operator product $\mathcal{O}_{q} \otimes \mathcal{O}_{q^{\prime}} \rightarrow \mathcal{O}_{q+q^{\prime}}$, as defined by the OPE in Eq. (1.11). All quantities are to be evaluated at the MIT in $d=2+\epsilon$.

We use a two-stage approach to the renormalization of the "extended" NL $\sigma \mathrm{M}$ [the action Eq. (2.7) supplemented with Eq. (2.27)]. The idea is to first locate the nontrivial metalinsulator fixed point in $d=2+\epsilon$, obtained via the standard $\varepsilon$ expansion by renormalizing the theory in Eq. (2.7) with $h$ $\rightarrow 0$. (We will use dimensional regularization.) We then compute the OPE [Eq. (1.11)] at the MIT to the lowest nontrivial order in $\sqrt{\epsilon}$. Finally, we run a "one-loop" RG calculation at this nontrivial fixed point for the full model defined by Eqs. (2.7) and (2.27). The required one-loop functional renormalization-group equation is obtained from the OPE. ${ }^{64}$ Note that since we are interested in LDOS and IPR statistics at the Anderson metal-insulator transition $\left(t=t^{*}\right)$ rather than in the diffusive metallic phase $\left(t<t^{*}\right)$, we are required to run the FRG at this nontrivial fixed point. ${ }^{65}$

In order to streamline the presentation, the abovedescribed field theory calculations are relegated to the last Sec. V of this paper. The obtained results required for the functional RG are simply invoked as needed in the next Sec. III, so that the reader less interested in calculational details may avoid Sec. V entirely.

\section{FUNCTIONAL RG FOR THE TYPICAL $\tau(q)$ SPECTRUM}

\section{A. From coupled RG to KPP equations}

The typical $\tau(q)$ spectrum, defined in Sec. I by Eq. (1.3a), can be extracted from the generating function $F_{q}(\xi ; L)$, introduced in Eq. (2.4). The relationship is expressed by Eq. (2.6). In terms of the NL $\sigma \mathrm{M}$ formulation reviewed in Secs. II B-II D, $F_{q}(\xi ; L)$ may be encoded as

$$
F_{q}(\xi ; L) \sim\left\langle\exp \left[\sum_{p=1}^{\infty} \frac{(p q) !}{p !} y_{p q} \int d^{d} \mathbf{r} \llbracket \mathcal{O}_{p q} \rrbracket(\mathbf{r})\right]\right\rangle,
$$

where $q=1,2,3, \ldots$ and $\llbracket \mathcal{O}_{p q} \rrbracket(\mathbf{r})$ is a "renormalized and normalized" LDOS moment eigenoperator, defined by Eq. (5.35) in the technical Sec. V of this paper. Note that here $p q$ denotes the product of the integers $p$ and $q . \llbracket \mathcal{O}_{m} \rrbracket(\mathbf{r})$ is just a normalized version of the LDOS moment operator $\mathcal{O}_{m}(\mathbf{r})$, defined previously via Eq. (2.24). [The careful (RG schemedependent) normalization of operators is an important technical step required for the accurate computation of correlation functions at the MIT, as detailed in Sec. V. In this section, we merely assert that the proper procedure has been implemented.] The factor $(p q) ! / p$ ! in Eq. (3.1) sets the normalization of the coupling constant $y_{p q}{ }^{66}$

Equation (3.1) generalizes Eq. (2.27) for the case of $q$ $>1$; in order to compute $F_{q}(\xi ; L)$, one must augment the bare sigma model action with the operator tower $\left\{\llbracket \mathcal{O}_{q} \rrbracket, \llbracket \mathcal{O}_{2 q} \rrbracket, \llbracket \mathcal{O}_{3 q} \rrbracket, \ldots\right\}$, since through the OPE operators representing lower integral LDOS moments generate new ones representing higher integral multiples of these. For $q$ $>1$, the operators in Eq. (3.1) form a subset of those in Eq. (2.27). The expectation $\langle\cdots\rangle$ in Eq. (3.1) is taken with respect to the NL $\sigma \mathrm{M}$ action at the MIT in $d=2+\epsilon$, Eq. (2.7), with $h=0$ and $t=t^{*}$. The coupling constants $\left\{y_{p q}\right\}$ take the bare values

$$
y_{p q}(l=0)=-\frac{\xi}{q !} \delta_{p, 1} .
$$

Here, $l=\ln L / L_{0}$ is the $\log$ of the spatial length scale $L$ (e.g., the system size), with $L_{0}$ an arbitrary reference scale.

A simple, straightforward approach to computing $F_{q}(\xi ; L)$ is the cumulant expansion of Eq. (3.1), evaluated at $L=L_{0}$ [i.e. using the bare coupling constants in Eq. (3.2)],

$$
\begin{aligned}
F_{q}\left(\xi ; L_{0}\right) \sim & \exp \left[-\xi \int d^{d} \mathbf{r}\left\langle\llbracket \mathcal{O}_{q}(\mathbf{r}) \rrbracket\right\rangle\right. \\
& \left.+\frac{\xi^{2}}{2 !} \int d^{d} \mathbf{r} d^{d} \mathbf{r}^{\prime}\left\langle\llbracket \mathcal{O}_{q}(\mathbf{r}) \rrbracket \llbracket \mathcal{O}_{q}\left(\mathbf{r}^{\prime}\right) \rrbracket\right\rangle_{c}+\cdots\right],
\end{aligned}
$$

where $\left\langle\mathcal{O}_{1} \mathcal{O}_{2}\right\rangle_{c} \equiv\left\langle\mathcal{O}_{1} \mathcal{O}_{2}\right\rangle-\left\langle\mathcal{O}_{1}\right\rangle\left\langle\mathcal{O}_{2}\right\rangle$, etc. To the first order in the expansion, 


$$
F_{q}\left(\xi ; L_{0}\right) \sim \exp \left(-\xi L_{0}^{d-x_{q}^{*}}\right)
$$

In this equation, $x_{q}^{*}$ denotes the negative scaling dimension of the operator $\llbracket \mathcal{O}_{q} \rrbracket(\mathbf{r})$ at the MIT; for the unitary class studied here, the result to lowest order in $\sqrt{\epsilon}$ was given by Eqs. (1.6a) and (1.6c) above. Combining Eqs. (3.4) and (2.6), we immediately recover Eq. (1.4): the lowest-order cumulant approximation to $F_{q}\left(\xi ; L_{0}\right)$ equates the typical $\tau(q)$ spectrum with $\tilde{\tau}(q)$ [Eqs. (1.5)-(1.6c)], associated to the average of the IPR. For sufficiently large LDOS moments with $q>q_{c}$, where $q_{c} \gg 1$ for perturbatively accessible $\epsilon \ll 1$ [Eq. (1.10)], this identification must break down (see the discussion in Sec. I A); an accurate computation of $F_{q}\left(\xi ; L_{0}\right)$ then appears to require the retention of higher-order cumulants.

In fact, the cumulant expansion fails to converge for any integral $q>1$, i.e. for both $q>q_{c}$ and $q_{c} \geq q>1$. The source of the problem is easily identified in Eq. (3.3), where one immediately sees the need for the OPE, as defined by Eq. (1.11). The second and higher cumulants in Eq. (3.3) involve products of LDOS moment operators, integrated over the sample volume. When two (or more) such operators approach the same spatial position, fusion can occur, in which new, higher moment operators are generated through shortdistance regularization. ${ }^{28-30,60,64}$ At the MIT, the negative scaling dimensions $\left\{x_{q}^{*}\right\}$ of the LDOS moment operators [Eqs. (1.6a), (1.6b), and (1.6c)] satisfy the convexity relation given by Eq. (1.12). Therefore, operators corresponding to successively higher moments $\left\{\llbracket \mathcal{O}_{2 q} \rrbracket, \llbracket \mathcal{O}_{3 q} \rrbracket, \ldots\right\}$ carry ever more negative scaling dimensions and these produce ever larger contributions to the cumulant expansion, even for moderate $q$ such that $1<q<q_{c}$. We therefore require an alternative calculational strategy which simultaneously incorporates fusion processes to all orders.

Rather than compute the generating function $F_{q}(\xi ; L)$ directly, we will use scaling arguments to extract its asymptotic behavior in the large system size limit, $L / L_{0} \rightarrow \infty$. In Eq. (3.1), the LDOS moment operators $\left\{\llbracket \mathcal{O}_{p q} \rrbracket\right\}$ perturb the action of the critical field theory. It is well known that the lowestorder RG equations for the set of conjugate coupling constants $\left\{y_{p q}\right\}$ follow directly from the operator product expansion. ${ }^{64}$

In Sec. V, we demonstrate that (the properly normalized versions of) the operators defined by Eq. (2.24) obey the OPE given by Eq. (1.11) at the MIT in $d=2+\epsilon$. We find that the OPE coefficient is given by the "tree level" (zero coupling) amplitude

$$
C_{q, q^{\prime}}^{q+q^{\prime}}=\frac{\left(q+q^{\prime}\right) !}{q ! q^{\prime} !}+\boldsymbol{O}(\boldsymbol{\epsilon})
$$

These results are obtained as Eqs. (5.33) and (5.34) in Sec. V, where we demonstrate that the lowest-order $t^{*} \propto \sqrt{\epsilon}$ (oneloop) correction to the OPE coefficient in Eq. (3.5) vanishes. Using Eqs. (1.11) and (3.5), one finds the infinite set of RG equations ${ }^{64}$

$$
\frac{d y_{p q}}{d l}=\left(d-x_{p q}^{*}\right) y_{p q}+\frac{S_{d}}{2} \sum_{m=1}^{p-1}\left(\begin{array}{c}
p \\
m
\end{array}\right) y_{m q} y_{(p-m) q}+\boldsymbol{O}\left(y^{3}\right),
$$

where $S_{d}$ is the surface area of the sphere in $d$ dimensions. Through the OPE, lower moment coupling constants always generate higher ones; the convexity property in Eq. (1.12) implies that, for $p>p^{\prime}$, a nonzero $y_{p q}$ represents a much more relevant perturbation than $y_{p^{\prime} q}$ to the critical NL $\sigma \mathrm{M}$ action. Clearly we must retain the entire infinite set $\left\{y_{p q}\right\}$ in our analysis.

At first glance, the generation of infinitely many relevant couplings would seem to imply nonuniversality: there are infinitely many classes of solutions to the RG equations (3.6) and hence there are infinitely many ways to depart from the $\mathrm{RG}$ fixed point representing the MIT. This is consistent with the fact that a random critical point should be characterized by the entire distribution functions of physical quantities, which can become very broad. At a delocalization critical point, however, the multifractal $\tau(q)$ and $f(\alpha)$ spectra, associated to a typical wave function in a fixed disorder realization, are both self-averaging ${ }^{16,24-26}$ and universal. 5,15,17,19,20 We will demonstrate that the FRG method gives a universal prediction for $\tau(q)$ and $f(\alpha)$, below and above termination (as defined in Sec. I A), at the unitary class Anderson MIT in $d=2+\epsilon$ consistent with this picture.

We can trade the coupled set of ordinary differential equations in Eq. (3.6) for a single partial differential equation (PDE) by defining the auxiliary generating function ${ }^{30}$

$$
G_{q}(z, l) \equiv \widetilde{G}_{q}(\widetilde{z}, \widetilde{l}) \equiv 1+\frac{S_{d}}{2 d} \sum_{p=1}^{\infty} \frac{\left(e^{-z}\right)^{p}}{p !} y_{p q}(l)
$$

where we have introduced the "position coordinate" $z$. $\widetilde{G}_{q}(\widetilde{z}, \widetilde{l})$ is a "Galilean boost" of $G_{q}(z, l)$ with

$$
\widetilde{z} \equiv z+\Xi q l, \quad \tilde{l} \equiv l .
$$

At the unitary class MIT in $d=2+\epsilon$, the parameter $\Xi$ has the value given by Eq. (1.6c) to one-loop order. The construction of $G_{q}(z, l)$ is motivated by the idea of a cumulant generating function for the LDOS moment operator $\llbracket \mathcal{O}_{q} \rrbracket$, imagining $y_{p q}$ to play the role of the $p$ th cumulant of $\llbracket \mathcal{O}_{q} \rrbracket$. At the same time, we stress that both $F_{q}(\zeta ; l)$ and $G_{q}(z, l)$ incorporate nonlocal correlations between LDOS moment operators, as mediated by the OPE.

Using the RG equations (3.6) for the coupling constants $\left\{y_{p q}\right\}$ and the explicit form of $x_{p q}^{*}$ from Eqs. (1.6a), (1.6b), and $(1.6 \mathrm{c})$, one can easily show ${ }^{30}$ that $\widetilde{G}_{q}(\widetilde{z}, \widetilde{l})$ satisfies the following KPP equation:

$$
\frac{1}{d} \partial_{l} \widetilde{G}_{q}=D_{q} \partial_{\widetilde{z}}^{2} \widetilde{G}_{q}+\widetilde{G}_{q}\left(\widetilde{G}_{q}-1\right)
$$

where we have introduced the effective diffusion constant 


$$
D_{q} \equiv \frac{q^{2} \Xi}{d}
$$

The same Eq. (3.9) was obtained in previous FRG studies of 2D disordered systems. ${ }^{28-30}$

\section{B. Solution to the KPP equation and results}

The KPP equation (3.9) describes nonlinear diffusion phenomena. The positive $D_{q}$ in Eq. (3.10) reflects the diffusion of the distribution function for the IPR, defined by Eq. (1.1). For high moments, $q \gg 1$, this diffusion constant is very large, indicating that the IPR becomes broadly distributed in the large system size limit; in this regime, the $\widetilde{\tau}(q)$ spectrum associated with the average IPR [Eq. (1.3b)] is dominated by rare realizations of the disorder and loses its meaning with respect to the typical wave function. The nonlinear term in Eq. (3.9) appears because the generating functions $\widetilde{G}_{q}(\widetilde{z}, \widetilde{l})$ and $F_{q}(\xi ; L)$ encode information about the typical $\tau(q)$ spectrum; this nonlinearity arises through the OPE between LDOS moment operators [Eqs. (1.11) and (3.5)]. As explained in the paragraph following Eq. (3.3), the OPE is the essential ingredient required in the computation of $\tau(q)$, which was missed in previous treatments ${ }^{8,44,45}$ of the Anderson MIT based on the NL $\sigma \mathrm{M}$ approach.

Nonlinear PDEs are often not analytically solvable, but a number of key results are known for the KPP equation. We summarize here only those features essential to the computation of $\tau(q)$; for further details, consult Refs. 28-30 and references therein. For a large class of initial conditions which satisfy

$$
\begin{aligned}
& \lim _{\tilde{z} \rightarrow+\infty} \tilde{G}_{q}(\widetilde{z}, 0)=1, \\
& \lim _{\tilde{z} \rightarrow-\infty} \tilde{G}_{q}(\widetilde{z}, 0)=0,
\end{aligned}
$$

$\widetilde{G}_{q}(\widetilde{z}, \widetilde{l})$ converges to a stable traveling-wave solution propagating in the positive $\widetilde{z}$ direction,

$$
\widetilde{G}_{q}(\tilde{z}, \tilde{l} \rightarrow \infty) \rightarrow h\left(\tilde{z}-\tilde{c}_{q} l\right),
$$

where the constant $\tilde{c}_{q}$ denotes the wave-front velocity. The functional form of the traveling wave in Eq. (3.12) is sensitive to the details of the initial condition at $\tilde{l}=0$. On the contrary, for an initial wave front satisfying the asymptotic property

$$
\tilde{G}_{q}(\widetilde{z} \rightarrow+\infty, 0) \sim 1-\lambda e^{-\widetilde{z}},
$$

with $\lambda$ a pure number, the velocity $\widetilde{c}_{q}$ is universal, depending only upon the diffusion constant $D_{q}$, defined in the context of the MIT by Eq. (3.10) above. Note that Eq. (3.13) constrains $\widetilde{G}_{q}$ only in the region penetrated by the wave front [Eq. (3.12)] in the limit of large "renormalization time," $\tilde{l} \rightarrow \infty$. Remarkably, the wave-front velocity is also insensitive to the precise form of the nonlinear term $\mathcal{F}\left(\widetilde{G}_{q}\right) \equiv \widetilde{G}_{q}\left(\widetilde{G}_{q}-1\right)$ in the KPP equation (3.9). In fact, the same velocity obtains from KPP for any nonlinear forcing function satisfying the constraints

$$
\begin{aligned}
& \mathcal{F}(0)=\mathcal{F}(1)=0, \quad \mathcal{F}(\widetilde{G})<0, \\
& \frac{d \mathcal{F}(\tilde{G})}{d \tilde{G}} \geq-1, \quad \frac{d \mathcal{F}(\tilde{0})}{d \widetilde{G}}=-1
\end{aligned}
$$

for $0 \leq \widetilde{G} \leq 1$. In this sense, the KPP equation achieves a strong version of universality.

Let us now return to the problem at hand, computing the typical $\tau(q)$ spectrum obtained at the MIT in the unitary class for $d=2+\epsilon$. The initial condition for the KPP Eq. (3.9) implied by Eq. (3.2) is

$$
\widetilde{G}_{q}(\widetilde{z}, 0)=1-\frac{\xi}{q !} \frac{S_{d}}{2 d} e^{-\widetilde{z}},
$$

consistent with only $y_{q}$ nonvanishing. Equation (3.15) satisfies the condition in Eq. (3.11a), having the same form as that expressed in Eq. (3.13). In order to satisfy Eq. (3.11b), we must bind the amplitude $0 \leq \widetilde{G}_{q}(\widetilde{z} \rightarrow-\infty, 0) \leq 1$; to that end, we deform Eq. (3.2) as follows:

$$
\begin{gathered}
y_{q}(0)=-\frac{\xi}{q !}, \\
y_{p q}(0) \rightarrow\left(\frac{S_{d}}{2 d}\right)^{p-1}\left[y_{q}(0)\right]^{p},
\end{gathered}
$$

which leads to

$$
\widetilde{G}_{q}(\widetilde{z}, 0) \sim \exp \left[-\frac{\xi}{q !} \frac{S_{d}}{2 d} e^{-\widetilde{z}}\right] .
$$

Crucially, since Eq. (3.17) satisfies Eq. (3.13), the asymptotic traveling-wave velocity $\widetilde{c}_{q}$ [Eq. (3.12)] depends only upon the diffusion constant $D_{q}$, Eq. (3.10). For the KPP equation (3.9) satisfying Eqs. (3.11a), (3.11b), and (3.13), one finds qualitatively different behavior for $D_{q}$ less than or greater than 1 (Refs. 28-30):

$$
\tilde{c}_{q}= \begin{cases}d\left(1+D_{q}\right), & D_{q} \leq 1 \\ 2 d \sqrt{D_{q}}, & D_{q}>1,\end{cases}
$$

Reversing the Galilean boost in Eq. (3.12) via Eq. (3.8), we see that

$$
\begin{gathered}
G_{q}(z, l \rightarrow \infty) \sim h\left(z-c_{q} l\right), \\
c_{q} \equiv \widetilde{c}_{q}-\Xi q .
\end{gathered}
$$

Let us try to understand the physics implied by Eq. (3.19). The generating function $G_{q}(z, l)$ was defined via Eq. (3.7) in terms of the infinite tower of coupling constants $\left\{y_{p q}\right\}$; the latter were introduced in the $\mathrm{NL} \sigma \mathrm{M}$ definition of $F_{q}(\xi ; L)$, Eq. (3.1). Under a change of length scale (e.g. an incremental increase in the sample size), each $y_{p q}$ evolves according to the RG Eq. (3.6). If we neglect the nonlinear terms in this equation due to the OPE, then each nonzero $y_{p q}$ grows under renormalization according to its own scaling exponent $c_{p q}^{(0)}$ $\equiv d-x_{p q}^{*}$; a full characterization of the system requires the specification of the entire set $\left\{c_{p q}^{(0)}\right\}, p \in \mathbb{N}$. As argued in Sec. 
I and below Eq. (3.6), we nevertheless expect that a single, well-defined exponent $\tau(q)$ can be defined for the IPR associated to a typical wave function even in the limit of relatively "large" $q$. Equation (3.19) implies that, through the OPE and the subsequent nonlinearity of the KPP equation, the functional RG proves this assertion: the asymptotic scaling of $G_{q}(z, l)$ involves a single number, the velocity $c_{q}$ given by Eqs. (3.18) and (3.19), which we can think of as a "typical" scaling exponent.

Since $G_{q}(z, l)$ tracks the scaling of coupling constants, we infer that the set $\left\{y_{p q}\right\}$ "fuses" into a single, typical coupling $y_{q}^{\text {typ }}$, up to less relevant perturbations to the critical NL $\sigma \mathrm{M}$ fixed point; we can then define an associated typical anomalous dimension

$$
\begin{aligned}
x_{q}^{\mathrm{typ}} & \equiv d-c_{q} \\
& = \begin{cases}-\Xi q(q-1), & 1 \leq q \leq q_{c} \\
d(1-q)+q(\sqrt{d}-\operatorname{sgn}(q) \sqrt{\Xi})^{2}, & q>q_{c},\end{cases}
\end{aligned}
$$

where we have used Eq. (3.10). In this equation, the critical value of $q_{c}$ corresponds to $D_{q}=1$, defined previously in Sec. I by Eq. (1.10).

We infer from Eq. (3.20) that $F_{q}(\xi ; L)$ acquires the following asymptotic form:

$$
\begin{aligned}
F_{q}(\xi ; L \rightarrow \infty) & \sim\left\langle\exp \left[y_{q}^{\mathrm{typ}} \int d^{d} \mathbf{r} \llbracket \mathcal{O}_{q}^{\mathrm{typ}} \rrbracket(\mathbf{r})\right]\right\rangle \\
& \sim \exp \left(y_{q}^{\mathrm{typ}} L^{d-x_{q}^{\mathrm{typ}}}\right) .
\end{aligned}
$$

As in Eq. (3.4), we have evaluated $F_{q}(\xi ; L)$ in the lowestorder cumulant expansion; the crucial difference between Eqs. (3.3) and (3.4) and Eq. (3.21) resides in the implied order of operations. To obtain the final result in Eq. (3.21), we first coarse grain the system, say, by integrating-out short-wavelength degrees of freedom (in a Wilsonian picture). The coarse graining generates higher-order couplings $\left\{y_{p q}\right\}, p>1$, through the nonlinear RG Eq. (3.6). In the large system size $\operatorname{limit} l=\ln \left(L / L_{0}\right) \rightarrow \infty$, a single, well-defined typical coupling $y_{q}^{\text {typ }}$ emerges, associated to a new local operator $\llbracket \mathcal{O}_{q}^{\text {typ }} \rrbracket(\mathbf{r})$, whose scaling dimension is given by Eq. (3.20). Finally, we evaluate $F_{q}(\xi ; L \rightarrow \infty)$ to lowest order in the cumulant expansion, which gives Eq. (3.21). This is expected to be a correct representation of the asymptotic scaling limit because the functional RG has already built all of the most relevant operator "fusions" into the definition of $\llbracket \mathcal{O}_{q}^{\text {typ }} \rrbracket(\mathbf{r})$. The emergence of the associated $y_{q}^{\text {typ }}$ and $x_{q}^{\text {typ }}$ has been proven above using the properties of the KPP equation, Eq. (3.9).

Finally, we extract the typical $\tau(q)$ spectrum. As obtained in the limit of large but finite renormalization, the typical coupling $y_{q}^{\text {typ }}$ should have an analytic expansion in powers of the parameter $\xi$ [cf. Eqs. (3.2) and (3.16)]. Up to an irrelevant rescaling, we may write

$$
y_{q}^{\mathrm{typ}}=-\xi-\sum_{m=2}^{\infty} \mathcal{Y}_{q m}^{\mathrm{typ}} \xi^{m} .
$$

Combining Eqs. (2.6) and (3.21), we obtain

$$
\tau(q) \sim \frac{d}{d \ln L} \int_{0}^{\infty} \frac{d \xi}{\xi}\left[e^{y^{\mathrm{typ}} L^{d-x_{q}^{\mathrm{typ}}}}-q e^{y_{1}^{\mathrm{typ}} L^{d-x_{1}^{\mathrm{typ}}}}\right] .
$$

In the limit $L \rightarrow \infty$, we may neglect all but the first term in Eq. (3.22), since $d-x_{q}^{\mathrm{typ}} \geq 0$ for all $q \geq 1$, provided $\Xi \leq 4 d$. This condition is always satisfied in the perturbatively accessible regime, $0<\epsilon \ll 1$, where the parameter $\Xi=\sqrt{\epsilon / 2}$ $+\boldsymbol{O}(\epsilon) \ll 1$ [Eq. (1.6c)]. Then we obtain using Eq. (2.5)

$$
\tau(q)=d(q-1)+x_{q}^{\mathrm{typ}}-q x_{1}^{\mathrm{typ}} .
$$

Equation (3.24) for the typical $\tau(q)$ should be compared to Eq. (1.5) for $\tilde{\tau}(q)$. In the perturbative regime $\epsilon \ll 1$, we have $x_{1}^{\text {typ }}=0$ [Eq. (3.20)]. Combining Eqs. (3.20) and (3.24), we arrive at our final result, the typical $\tau(q)$ spectrum given by

$$
\tau(q)= \begin{cases}d(q-1)\left(1-\frac{q}{q_{c}^{2}}\right), & |q| \leq q_{c} \\ d\left(1-\frac{\operatorname{sgn}(q)}{q_{c}}\right)^{2} q, & |q|>q_{c},\end{cases}
$$

where $q_{c}=\sqrt{d / \Xi}$ [Eq. (1.10)]. In this equation, we have extended $q$ from the positive integers to the entire real line (see also Ref. 53). The average $\widetilde{\tau}(q)$ and typical $\tau(q)$ spectra are respectively sketched in the top and bottom panels of Fig. 1 in Sec. I.

The singularity spectrum $f(\alpha)$ was introduced in Eq. (1.2). The $f(\alpha)$ corresponding to the typical $\tau(q)$ in Eq. (3.25), as obtained from the Legendre transformation, is

$$
f(\alpha)= \begin{cases}d-\frac{(\alpha-d-\Xi)^{2}}{4 \Xi} & \\ =\frac{q_{c}^{2}\left(\alpha_{+}-\alpha\right)\left(\alpha-\alpha_{-}\right)}{4 d}, & \alpha_{-} \leq \alpha \leq \alpha_{+} \\ 0, & \alpha<\alpha_{-}, \alpha_{+}<\alpha .\end{cases}
$$

The spectral cutoffs $\alpha_{ \pm}$were defined by Eq. (1.9). As expected, $f(\alpha)$ associated to the typical wave function is never negative, as discussed in Sec. I (see also the top and bottom panel insets in Fig. 1). Equations (3.25) and (3.26) hold to the lowest nontrivial order in $\sqrt{\epsilon}$. The consistency of the restriction to only the lowest-order contributions in the $\epsilon$ expansion is demonstrated in Sec. IV.

An alternative representation of multifractality invokes the "generalized dimension" $D_{q}$, defined via

$$
\tau(q) \equiv(q-1) D_{q}
$$

Spectral termination [Eqs. (3.25) and (3.26)] implies that $\alpha_{+} \leq D_{q} \leq \alpha_{-}$, with the boundary values associated to the limits

$$
\lim _{q \rightarrow \pm \infty} D_{q}=\alpha_{\mp}
$$

Numerical computations of $D_{q}$ for the typical wave function confirm Eq. (3.28) (see, e.g., Refs. 14 and 15). 


\section{Comparison to other arguments}

Our final results (3.25) and (3.26) agree with previous heuristic arguments given in Refs. 16, 25, and 38. As explained below Eq. (1.2), $f(\alpha)$ describes the measure $L^{f(\alpha)}$ of the set of those points $\mathbf{r}$ where the eigenfunction $\psi$ takes the value $|\psi(\mathbf{r})|^{2} \propto L^{-\alpha} \cdot{ }^{10}$ Hence, the IPR $P_{q}[$ Eq. (1.1)] can be estimated as an integral

$$
P_{q} \sim \int_{f(\alpha) \geq 0} d \alpha L^{-q \alpha+f(\alpha)} .
$$

The integrand takes a maximum value at a saddle-point value $\alpha$, which defines the $\tau(q)$. For $|q|<q_{c}$, this saddle point is in the integration domain, whereas for $|q|>q_{c}$, it is outside of it. In the latter case, the integral is dominated by the boundary value of $\alpha=\alpha_{\bar{\mp}}$, where $f\left(\alpha_{\overline{+}}\right)=0$.

\section{DISCUSSION}

We have provided a field theoretical description of the termination of the multifractal spectrum $\tau(q)$, as defined for the typical wave functions, at the Anderson MIT in $d=2+\epsilon$ for the unitary-disordered metal class. The essential ingredients of the calculation are evident in the formulation of Eqs. (2.6) and (3.1): these are the infinite set of properly normalized LDOS moment operators $\left\{\llbracket \mathcal{O}_{p q} \rrbracket(\mathbf{r})\right\}, p \in \mathbb{N}$, characterized by the negative scaling dimensions $\left\{x_{p q}^{*}\right\}$ in Eqs. (1.6a), (1.6b), and (1.6c). Each successive higher-moment operator with $p=\{1,2, \ldots\}$ constitutes a more strongly relevant perturbation to the critical RG fixed point that describes the MIT. Through the OPE [Eq. (1.11)], lower moments always generate higher ones and a consistent treatment of the problem requires that the entire infinite hierarchy of LDOS moment operators is retained. Ordinarily, the advent of an infinity of relevant scaling directions should cast serious doubt upon the adequacy of single (or few) parameter scaling, at least with respect to the investigated critical point; remarkably, the FRG "absorbs" the entire LDOS moment tower and through the (universal) properties of the long-time asymptotics of the KPP equation, renders in the end a single, universal prediction for the typical $\tau(q)$.

Physically, the relevant LDOS moments reflect the fact that a random critical point should be characterized by the distribution functions of physical quantities rather than their mean, variance, or first few moments. The distribution of an observable in the presence of quenched disorder can become very broad due to the influence of rare events. ${ }^{44,45,50-52}$ In principle, we need the FRG to obtain scaling for the entire probability distribution. ${ }^{67}$ For large $q$, the IPR, defined by Eq. (1.1) [or of its field-theoretic generalization, Eq. (2.3)], constitutes such a broadly distributed observable. , $^{2,16,24,25,31}$ By comparison, a universal $\tau(q)$ spectrum for the typical wave function obtains because the log of the IPR is selfaveraging for all $q \cdot{ }^{16,24-26}$

Technically, the FRG method implemented in Sec. III is completely analogous to that employed previously $y^{28-30}$ in the study of certain special 2D disordered field theories, possessing an additional, "chiral" symmetry. ${ }^{55}$ (See the end of Sec. I B for a description of these chiral models.) As in this prior work, the FRG translates the infinite set of coupled flow equations (3.6) into the KPP Eq. (3.9) for a certain (auxiliary) generating function. Through the asymptotic solution of the KPP equation in the form of a propagating wave front, the tower of relevant LDOS moment operators combines via multiple OPEs into a single, typical operator (up to less relevant perturbations), characterized by the typical scaling dimension in Eq. (3.20). The final results for $\tau(q)$ and $f(\alpha)$ [Eqs. (3.25) and (3.26)] are obtained via the FRG for the unitary universality class, using only two inputs, evaluated at the MIT: (i) the scaling dimensions $\left\{x_{q}^{*}\right\}$ (associated to the average operator scaling, already known from previous work) (Refs. 8 and 34-36) and (ii) the OPE coefficient $C_{q, q^{\prime}}^{q+q^{\prime}}$, Eq. (3.5) (computed in Sec. V to lowest nontrivial order in $\sqrt{\epsilon}$ ). The former is specific to the unitary class, but we have shown that the latter takes exactly the same form in the chiral model calculations. ${ }^{28-30}$

In treating the unitary class, we have chosen to work only to the lowest order in $t^{*} \propto \sqrt{\epsilon}$, i.e. to one loop. To this order, the resulting singularity spectrum $f(\alpha)$ given by Eq. (3.26) is purely quadratic over the region $\alpha_{+}<\alpha<\alpha_{-}$(the so-called "parabolic approximation"). ${ }^{4,5,18-20}$ We now discuss the consistency of working with the functional renormalization group to this order in the $\epsilon$ expansion. Corrections to the LDOS moment scaling dimensions $\left\{x_{q}^{*}\right\}$ are already known to four loops, ${ }^{35,36}$

$$
x_{q}^{*}=-\sqrt{\frac{\boldsymbol{\epsilon}}{2}} q(q-1)-\frac{3 \zeta(3)}{8} \epsilon^{2} q^{2}(q-1)^{2}+\boldsymbol{O}\left(\boldsymbol{\epsilon}^{5 / 2}\right),
$$

where $\zeta(z)$ denotes the Riemann zeta function. [Equation (4.1) implies that the quantity $x_{q}^{*} / q$ possesses an expansion in the parameter $q \sqrt{\epsilon}$, with coefficients that are analytic functions of $\sqrt{\epsilon}$.] While the FRG method formally retains LDOS moment operators $\left\{\llbracket \mathcal{O}_{q} \rrbracket(\mathbf{r})\right\}$ to arbitrarily high orders in $q$, it is crucial to note that the termination of the typical $\tau(q)$ spectrum [Eq. (3.25)] occurs at the finite value $q=q_{c}$ [Eq. (1.10)]; to lowest order,

$$
q_{c}^{2}=2 \sqrt{2 / \epsilon}+\boldsymbol{O}(1) .
$$

Evaluating the four-loop scaling dimension in Eq. (4.1) at $q_{c}$, we obtain

$$
x_{q=q_{c}}^{*}=-2+(2 \epsilon)^{1 / 4}+\boldsymbol{O}\left(\epsilon^{1 / 2}\right) .
$$

The one-loop approximation consists of retaining only the first term on the RHS of Eq. (4.1), as well as the terms written explicitly on the RHS of each of Eqs. (4.2) and (4.3). At termination $\left(q=q_{c}\right)$, the higher-order loop corrections give rise to additional terms in Eq. (4.3) that are down by higher powers of $\epsilon^{1 / 4}$ and these can be consistently neglected for $\epsilon \ll 1$.

We cannot resist contemplating, at a very speculative level, a naive extrapolation of our one-loop results to moderate or even large $\epsilon$. First, note that Eq. (3.20) implies the existence of a nonzero, typical scaling dimension $x_{1}^{\text {typ }}$ for the first moment of the LDOS, when $q_{c}<1$, 


$$
x_{1}^{\mathrm{typ}}=d\left(1-\frac{1}{q_{c}}\right)^{2}, \quad q_{c}<1 .
$$

Using the lowest-order result in Eq. (4.2), we then define

$$
\epsilon_{F} \equiv \epsilon\left(q_{c}=1\right) \sim 8 .
$$

At face value, a $x_{1}^{\mathrm{typ}}>0$ would imply that the typical LDOS vanishes at the MIT. Equation (4.5) suggests that this becomes possible in the limit of large spatial dimensionality, $\epsilon>\epsilon_{F}$. Such a scenario does not contradict rigorous results ${ }^{46,47}$ which prove that the average, global density of state (DOS) remains uncritical (constant) across the transition for any spatial dimension $d$. Indeed, Bethe lattice computations $^{6,68}$ exhibit a typical LDOS that vanishes exponentially across the transition; ${ }^{69}$ see also Refs. 70 and 71 . As the Bethe lattice can be equated with the limit of infinite spatial dimensionality, ${ }^{3}$ this picture in fact appears consistent with Eqs. (4.4) and (4.5) in the limit $\epsilon \rightarrow \infty$, where upon $x_{1}^{\text {typ }} \rightarrow \infty$.

It has been asserted ${ }^{3,72}$ that for Anderson localization, the upper critical dimension $d_{u c}=\infty$, i.e., the Bethe lattice case. For example, the arguments given in Ref. 72 were based in part upon the $\epsilon \rightarrow \infty$ limit of the one-loop multifractal spectrum $^{8,9}$ at the Anderson transition [in the time-reversal invariant (TRI) orthogonal class] in $d=2+\epsilon$; however, this work failed to correctly distinguish the average $\widetilde{\tau}(q)$ from the typical $\tau(q)$ spectra. The naive extrapolation of the results of the present work to large $\epsilon$ suggests an alternate possibility. To motivate the basic underlying idea, we note that for $\epsilon$ $>\epsilon_{F}$, the typical multifractal spectrum $\tau(q)$ defined by Eqs. (3.20) and (3.24) would take the form

$$
\tau(q)= \begin{cases}-d\left(1-\frac{q}{q_{c}}\right)^{2}, & |q| \leq q_{c} \\ \frac{2 d}{q_{c}}(q-|q|), & |q|>q_{c} .\end{cases}
$$

This should be contrasted with Eq. (3.25), which assumed $q_{c}>1$ [always the case in the perturbatively accessible regime, $0<\epsilon \ll 1-$ see Eq. (4.2)]. Equation (4.6) shows that $\tau(q)=0$ for all $q \geq q_{c}$ when $q_{c}<1 \quad\left(\epsilon>\epsilon_{F}\right)$. Thus the $\tau(q)$ spectrum "freezes" for dimensionalities above the threshold $d_{F} \equiv 2+\epsilon_{F}$. An analogous freezing transition has been predicted $^{24,28-30,38,39}$ for the 2D chiral Dirac models discussed at the end of Sec. I B; in these models, the transition occurs for quenched disorder fluctuation strengths larger than some threshold values. Unlike the unitary metal class discussed here, the chiral model freezing transition has been rigorously derived through strong randomness ${ }^{39}$ and FRG arguments, ${ }^{26,28-30}$ which do not rely upon expansion in a small parameter. By comparison, Eqs. (4.4) and (4.6) lie well beyond the perturbatively accessible regime. Regardless, the freezing scenario suggests the intriguing possibility of a finite $d_{F}<\infty$ for Anderson localization in the normal-metal classes, which could perhaps serve as a finite upper critical dimension. The existence of $d_{F}$ beyond the epsilon expansion and its relation (if any) to $d_{u c}$ are questions for future work. (a)

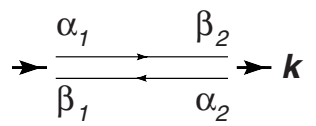

(b)

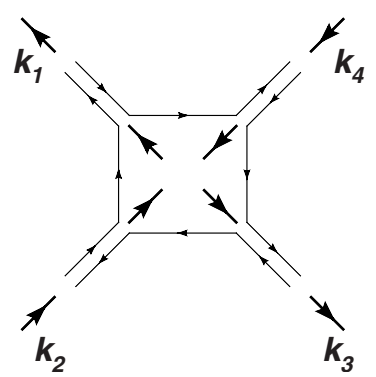

FIG. 2. (a) Propagator and (b) lowest-order vertex necessary for the one-loop RG, obtained by expanding Eq. (2.7) in terms of the unconstrained $\hat{W}$ field, using Eq. (2.10). Associated amplitudes are given by Eqs. (5.1) and (5.2) in the text.

\section{OPERATOR PRODUCT EXPANSION AT THE ANDERSON FIXED POINT: PERTURBATIVE CALCULATION}

In this final (technical) section, we provide a derivation of the LDOS moment operator algebra required for the functional RG construction in Sec. III. Using the NL $\sigma \mathrm{M}$ framework reviewed in Sec. II, we first rederive the anomalous scaling dimensions of the LDOS moment operators introduced in Sec. II C. We then turn to the perturbative evaluation of the operator product expansion, as defined by Eq. (1.11), between properly normalized versions of these eigenoperators.

\section{A. Renormalization of the model}

To begin, we consider the renormalization of the bare NL $\sigma \mathrm{M}$ defined by Eq. (2.7). This calculation is standard: ${ }^{1,43,73}$ we provide only our conventions necessary to set up the computation and the corresponding results. Using the parameterization in Eq. (2.10), one obtains the $\hat{W} \rightarrow W_{\beta}^{\alpha}$ field propagator and vertex shown in Fig. 2. The propagator is pictured in Fig. 2(a) as pair of counterdirected thin lines, representing physically an ambulating electron-hole pair ("diffuson") and mathematically the linking of direct and conjugate indices in two inequivalent representations of $U(n)$ [since the maximum compact subgroup of $U(2 n)$ is $U(n)$ $\times U(n)]$. Equivalently, in the unitary class with this parameterization, the field $W_{\beta}^{\alpha}$ is Wick-contracted only with its adjoint $W_{\alpha}^{\dagger \beta}$; this fact is indicated by the thick arrows in Fig. 2 , which also encode the direction of momentum flow. The amplitude corresponding to the propagator in Fig. 2(a) is

$$
\left\langle W_{\beta_{1}}^{\alpha_{1}}(\mathbf{k}) W_{\beta_{2}}^{\dagger \alpha_{2}}(\mathbf{k})\right\rangle=\delta_{\beta_{2}}^{\alpha_{1}} \delta_{\beta_{1}}^{\alpha_{2}} \frac{t_{0}}{|\mathbf{k}|^{2}+h_{0} t_{0}},
$$

where $t_{0}$ and $h_{0}$ are bare parameters. In this paper, we will only need the lowest-order nonlinear vertex $\equiv \mathfrak{V}_{4}$ [obtained via an expansion of Eq. (2.7) in powers of $\hat{W}]$; this vertex is pictured in Fig. 2(b), with the corresponding amplitude 


$$
\mathfrak{V}_{4}=\frac{2 !}{8 t_{0}}\left[\begin{array}{l}
2\left(\mathbf{k}_{1} \cdot \mathbf{k}_{3}+\mathbf{k}_{2} \cdot \mathbf{k}_{4}\right)-\mathbf{k}_{1} \cdot \mathbf{k}_{2} \\
-\mathbf{k}_{2} \cdot \mathbf{k}_{3}-\mathbf{k}_{3} \cdot \mathbf{k}_{4}-\mathbf{k}_{4} \cdot \mathbf{k}_{1}-2 h_{0} t_{0}
\end{array}\right] .
$$

Adopting standard dimensional regularization conventions, ${ }^{59,60,73}$

$$
\begin{aligned}
& t_{0} \equiv t \mu^{-\epsilon} F_{t}, \\
& h_{0} \equiv Z_{W}^{-1 / 2} h,
\end{aligned}
$$

with $t$ and $h$ the renormalized parameters, $\mu$ an arbitrary inverse-length scale, and $Z_{W}$ the field renormalization of the elementary operator $\hat{W}$. The RG flow equations are given by

$$
\begin{gathered}
\frac{d t}{d l}=\frac{-\epsilon t}{1+\frac{d \ln F_{t}}{d \ln t},} \\
\frac{d \ln h}{d l}=d+\frac{1}{2} \frac{d \ln Z_{W}}{d \ln t} \frac{d \ln t}{d l} .
\end{gathered}
$$

In Eqs. (5.3) and (5.4), $d=2+\epsilon$ is the spatial dimensionality of the system and $l \sim-\ln \mu$ is the logarithm of the spatial length scale.

For the compact unitary model with target space $U(2 n) / U(n) \times U(n)$, the one-loop flow equations are

$$
\begin{aligned}
& \frac{d t}{d l}=-\epsilon t+\frac{n t^{2}}{4 \pi}+\boldsymbol{O}\left(t^{3}\right), \\
& \frac{d \ln h}{d l}=d-\frac{n t}{4 \pi}+\boldsymbol{O}\left(t^{2}\right) .
\end{aligned}
$$

These equations possess a critical fixed point at the "temperature" $t^{*}=4 \pi \epsilon / n$, with $h=0$. The Anderson model corresponds to the limit $n \rightarrow 0$ in Eqs. (5.5) and (5.6); in this case, a nontrivial critical point occurs at two-loop order. ${ }^{1}$ In our conventions, the two-loop result is ${ }^{43,73}$

$$
\frac{d t}{d l}=-\epsilon t+\frac{t^{3}}{2^{5} \pi^{2}}+\boldsymbol{O}\left(t^{4}\right),
$$

valid in the limit $n \rightarrow 0$. The critical value of the inverse conductance at the metal-insulator transition in $d=2+\epsilon$ is proportional to $t^{*}=4 \pi \sqrt{2 \epsilon}+\boldsymbol{O}(\epsilon)$.

\section{B. Composite operator scaling dimensions}

Next, we turn to the renormalization of the composite operators defined by Eq. (2.23). The renormalization of $\mathcal{O}_{p\left[\beta_{1} \beta_{2} \cdots \beta_{p}\right]}^{\alpha_{1} \alpha_{2} \ldots \alpha_{p}}$ can be determined by considering "matrix elements" of that operator with arbitrary configurations of $p$ distant, mutually separated adjoint fields $\left\{W_{\gamma}^{\dagger \lambda}\right\}$. [For more general operators built out of products of both $\pi\left(\hat{W}, \hat{W}^{\dagger}\right)$ and $\sigma\left(\sqrt{\hat{\mathbb{I}}_{n}-\hat{W} \hat{W}^{\dagger}}, \sqrt{\hat{\mathbb{I}}_{n}-\hat{W}^{\dagger} \hat{W}}\right)$ components, one must typically consider multiple matrix element types involving different numbers of $\hat{W}$ and $\hat{W}^{\dagger}$ fields. $\left.{ }^{59}\right]$

Specifically, we define
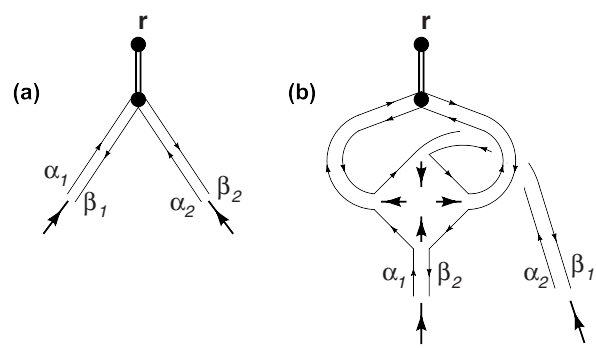

FIG. 3. Basic renormalization process of a composite operator.

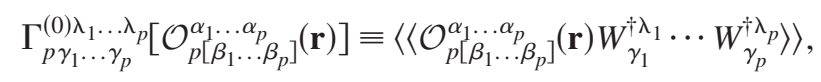

where the double angle brackets $\langle\langle\mathcal{O} \cdots\rangle\rangle$ signify the oneparticle irreducible matrix element of $\mathcal{O}$, amputating the external fields. ${ }^{60,74}$ The external $\left\{\hat{W}^{\dagger}\right\}$ fields are assumed to be located far from each other and from the position $\mathbf{r}$ of the composite operator. The superscript (0) on the left-hand side (LHS) of this equation indicates that this is a bare (i.e. not yet renormalized) quantity.

The basic one-loop process is illustrated in Fig. 3. Figure 3(a) depicts the two-field matrix element of the (unsymmetrized) operator

$$
W_{\beta_{1}}^{\alpha_{1}} W_{\beta_{2}}^{\alpha_{2}}(\mathbf{r}) .
$$

The vertex $\mathfrak{V}_{4}$ [Fig. 2(b) and Eq. (5.2)] pairwise permutes the lower indices of composite operator "legs," as shown in Fig. 3(b). The completely antisymmetrized operator defined by Eq. (2.23) is clearly an eigenoperator at one loop, since the sum of all diagrams to this order represents a complete symmetrization procedure. This is expected to hold to all higher orders in $t$ because $\mathcal{O}_{p\left[\beta_{1} \ldots \beta_{p}\right]}^{\left.\alpha_{1} \ldots \alpha_{p}\right]}$ plays the role of a "highest weight state" in an irreducible representation of the full $\mathrm{NL} \sigma \mathrm{M}$ target manifold symmetry group $U(2 n)$.

At one loop, the matrix element defined by Eq. (5.8) is equal to

$$
\left.\Gamma_{p \gamma_{1} \ldots \gamma_{p}}^{(0) \lambda_{1} \ldots \lambda_{p}}\left[\mathcal{O}_{p\left[\beta_{1} \ldots \beta_{p}\right]}^{\alpha_{1} \ldots \alpha_{p}}(\mathbf{r})\right] \sim\left[1-\frac{p(p-1)}{2} I_{1}\right] \mathfrak{A}_{\left.p \gamma_{1} \ldots \gamma_{p}: \beta_{1} \ldots \beta_{p}\right]}^{\lambda_{1} \ldots \lambda_{p} ; \alpha_{1} \ldots \alpha_{p}}\right]
$$

where $\mathfrak{A}_{p \gamma_{1} \ldots \lambda_{p} ;\left[\beta_{1} \ldots \beta_{p}\right]}^{\lambda_{1} \ldots \lambda_{p} ; \alpha_{1} \ldots \alpha_{p}}$ is the zeroth order amplitude [equal to zero or the pure constant $(1 / p !)^{2}$, depending upon the matrix element]. ${ }^{75}$ In order to save writing wherever possible, from this place forward, we will adopt the following shorthand notation: underlined vertices $(\underline{\Gamma})$, operators $(\mathcal{O})$, and tree level matrix elements $(\mathfrak{A})$ should be understood as possessing the appropriate set of indices and all indices in a given equation are matched (in the appropriate order). With these conventions established, Eq. (5.10) may be rewritten compactly as

$$
\underline{\Gamma}_{p}^{(0)}\left[\underline{\mathcal{O}}_{p}(\mathbf{r})\right] \sim\left[1-\frac{p(p-1)}{2} I_{1}\right] \underline{A}_{p} .
$$

In Eqs. (5.10) and (5.11), 


$$
I_{1}=\frac{-t_{0}}{2} \int \frac{d^{d} \mathbf{k}}{(2 \pi)^{d}} \frac{1}{|\mathbf{k}|^{2}+h_{0} t_{0}} \sim \frac{t}{4 \pi}\left[\frac{1}{\epsilon}+\frac{1}{2} \ln \left(\frac{h t e^{\gamma}}{4 \pi \mu^{2}}\right)\right]
$$

Here, we have used Eq. (5.3) and standard-dimensional regularization technology; $\gamma$ denotes the Euler-Mascheroni constant.

To renormalize Eq. (5.11), we insist that ${ }^{60}$

$$
Z_{p}^{-1} Z_{W}^{p / 2} \Gamma_{p}^{(0)}\left[\underline{\mathcal{O}}_{p}(\mathbf{r})\right]=\text { finite, }
$$

where $Z_{p}$ is the composite operator renormalization and the factor of $Z_{W}^{p / 2}$ compensates for the $p$ (amputated) external fields. One then obtains the scaling dimension

$$
x_{p}=\epsilon \frac{d \ln Z_{p}}{d \ln t}=\frac{t}{4 \pi}\left[n p-\frac{p(p-1)}{2}\right]+\boldsymbol{O}\left(t^{2}\right) .
$$

We make two observations. First, by evaluating Eq. (5.14) at the Anderson transition critical point located by $t^{*}$ $=4 \pi \sqrt{2 \epsilon}$, with $n \rightarrow 0$, we recover the known results ${ }^{34,36}$ for the multifractal spectrum $\widetilde{\tau}(p)$ associated to the averaged IPR, as provided above in Eqs. (1.5)-(1.6c). Second, we have only considered the renormalization of the fully antisymmetrized operator defined by Eq. (2.23) because this is the most relevant in the $n \rightarrow 0$ limit. The fully symmetrized operator $\mathcal{O}_{p\left(\beta_{1} \beta_{2} \ldots \beta_{p}\right)}^{\alpha_{1} \alpha_{2} \ldots \alpha_{p}}$, which is defined as in Eq. (2.23) without the $\operatorname{sgn}(\mathbf{P})$ factor in the summand, also constitutes an eigenoperator with scaling dimension

$$
x_{p(\mathrm{sym})}=\frac{t}{4 \pi}\left[n p+\frac{p(p-1)}{2}\right]+\boldsymbol{O}\left(t^{2}\right) .
$$

Consider the case of $n=1$. The fully antisymmetrized operator defined by Eq. (2.23) does not exist for $p>1$, since all $\hat{W} \Rightarrow W$ fields are scalars in this case. At the fixed point located by $t^{*}=4 \pi \epsilon / n$ with $n=1$, the symmetrized operator scaling dimension $x_{p(\mathrm{sym})}^{*}=\epsilon p(p+1) / 2+\boldsymbol{O}\left(\boldsymbol{\epsilon}^{2}\right)$, which is the expected result for spherical harmonic composite operators in the $U(2) / U(1) \times U(1) \sim O(3) / O(2) \mathrm{NL} \sigma \mathrm{M} .^{59}$

\section{Two-point function normalization at the MIT}

The set of coefficients $\left\{C_{q, q^{\prime}}^{q+q^{\prime}}\right\}$ [Eq. (1.11)] defining the OPE for properly normalized composite eigenoperators constitute universal numbers characterizing the MIT. The proper (RG scheme-dependent) normalization of each eigenoperator is such that its two-point autocorrelation function is schemeindependent at the critical point in $d=2+\epsilon .{ }^{64}$ In this subsection, we derive the normalization of the operators defined by Eq. (2.23) with respect to their two-point functions (at large spatial separation), while the OPE is considered in the sequel. Since we are interested in critical properties, we assume $h=0$ in Eq. (2.7) throughout the following discussion.

The technical tool for computing operator correlation functions at any perturbatively accessible fixed point is RGimproved perturbation theory (PT). In the case of the nontrivial NL $\sigma \mathrm{M}$ critical point in $d=2+\epsilon$, however, some technical difficulties arise. For a NL $\sigma \mathrm{M}$ with a compact, nonAbelian symmetry, the trivial fixed point located at $t=0$ is invariably infrared (IR) unstable in 2D. ${ }^{60}$ For any nonzero $t$, such a $2 \mathrm{D}$ model always flows under the RG toward a symmetry-restored, thermally disordered "paramagnetic" state. Renormalized perturbation theory at $t \ll 1$, for composite operator correlators that are not invariant under the full symmetry group of the sigma model target manifold, is typically plagued by IR divergences and hence affected by the specific way one regularizes these IR divergences.

The solution ${ }^{76-78}$ that we employ in this section is to consider only invariant correlation functions. Invariant correlators are free of IR divergences, and a sensible renormalized PT for these objects can be constructed. ${ }^{79}$ For the OPE in Sec. V D, we will see that this restriction is unnecessary.

We stress that, by the same token, all eigenoperators at the nontrivial fixed point in $d=2+\epsilon$ possess well-defined critical correlations. Thus the above-described calculational impasse, as well as its solution, in fact reflects peculiarities of the $\epsilon$ expansion rather than the NL $\sigma \mathrm{M}$ itself (at least for $d>2$ ).

Within the symmetry-broken phase, $\pi\left(\hat{W}, \hat{W}^{\dagger}\right)$ and $\sigma$ $\left(\sqrt{\hat{I}_{n}-\hat{W} \hat{W}^{\dagger}}, \sqrt{\hat{I}_{n}-\hat{W}^{\dagger} \hat{W}}\right)$ fields possess very different correlation functions: the former constitute Goldstone modes with massless correlations, while the latter are gapped longitudinal modes, with massive correlation functions for all $t<t^{*}$. At the critical point $t=t^{*}$ for $n>0$, symmetry is restored; here, all operators belonging to a given irreducible representation of the target manifold symmetry group will possess identical correlations, provided a group-invariant normalization is chosen for these operators. [For the $O(3) / O(2)$ model, an invariant normalization is that conventionally assigned to spherical harmonics, written in terms of $\pi$ and $\sigma$ coordinates.] We will use this fact to determine the two-point function normalization of $\mathcal{O}_{p\left[\beta_{1} \beta_{2} \ldots \beta_{p}\right]}^{\alpha_{1} \alpha_{2} \ldots \alpha_{p}}[$ Eq. (2.23)] for $n$ $=\{1,2, \ldots\}$ and then continue the result to $n \rightarrow 0$.

Consider the following invariant, "nonlocal" operator:

$$
\Omega_{p}\left(\mathbf{r}, \mathbf{r}^{\prime}\right) \equiv \sum_{\{m\}} \Theta_{p\{m\}}(\mathbf{r}) \Theta_{p\{m\}}^{*}\left(\mathbf{r}^{\prime}\right)
$$

where $\Theta_{p\{m\}}$ is a composite operator that is a component of an irreducible representation of the sigma model symmetry group. The representation is distinguished by the Casimir parameter $p$, while the component operators are labeled by a set of "magnetic" quantum numbers $\{m\}$, e.g. $\left\{\alpha_{1}, \ldots, \alpha_{p},\left[\beta_{1}, \ldots, \beta_{p}\right]\right\} \in\{m\}$ for the antisymmetrized LDOS moment operators defined by Eq. (2.23). One may use expressions for the $\Theta_{p\{m\}}$ in terms of the target manifold coordinates $\left(\hat{W}, \hat{W}^{\dagger}, \sqrt{\hat{\mathbb{I}}_{n}-\hat{W} \hat{W}^{\dagger}}, \sqrt{\hat{\mathbb{I}}_{n}-\hat{W}^{\dagger} \hat{W}}\right)$ in order to construct an explicit expression for the RHS of Eq. (5.16); at the nontrivial critical point in $d=2+\epsilon$, however, we require only the lowest-order expansion for the expectation of Eq. (5.16) in powers of $t^{*} \propto \epsilon^{\sigma}(\sigma=1$ or $1 / 2$ for $n \in \mathbb{N}$ or $n \rightarrow 0$, respectively). Since an expansion in powers of $t$ is equivalent to an expansion in powers of $\hat{W}$ and $\hat{W}^{\dagger}$, we make the following ansatz: 


$$
\begin{aligned}
\Omega_{p}\left(\mathbf{r}, \mathbf{r}^{\prime}\right) \sim & \widetilde{\alpha}_{p}\left(1+\frac{\alpha_{p, 1}}{2 n^{2}} \operatorname{Tr}\left[\hat{W}^{\dagger}(\mathbf{r}) \hat{W}\left(\mathbf{r}^{\prime}\right)-\hat{W}^{\dagger}(\mathbf{r}) \hat{W}(\mathbf{r})\right.\right. \\
& \left.\left.+\hat{W}^{\dagger}\left(\mathbf{r}^{\prime}\right) \hat{W}(\mathbf{r})-\hat{W}^{\dagger}\left(\mathbf{r}^{\prime}\right) \hat{W}\left(\mathbf{r}^{\prime}\right)\right]+\boldsymbol{O}\left(\hat{W}^{4}\right)\right) .
\end{aligned}
$$

The expectation of the assumed form of Eq. (5.17) is free from IR divergences. While the numerical coefficient $\widetilde{\alpha}_{p}$ in this equation is arbitrary, $\alpha_{p, 1}$ is not and can in principle be computed from knowledge of the representation theory of the NL $\sigma \mathrm{M}$ symmetry group; instead, we will determine its value empirically below. Note that Eq. (5.17) is manifestly invariant under $U(n) \times U(n)$ subgroup transformations, $\hat{W}$ $\rightarrow \hat{U}_{L} \hat{W} \hat{U}_{R}$, with $\hat{U}_{L / R}^{\dagger} \hat{U}_{L / R}=\hat{I}_{n}$.

We compute the expectation of Eq. (5.17) in position space $^{77}$ using the IR-convergent Green's function at zero coupling

$$
\begin{aligned}
& \left\langle W_{\beta_{1}}^{\dagger \alpha_{1}}(\mathbf{r}) W_{\beta_{2}}^{\alpha_{2}}(0)-W_{\beta_{1}}^{\dagger \alpha_{1}}(0) W_{\beta_{2}}^{\alpha_{2}}(0)\right\rangle_{0} \\
& \quad=\delta_{\beta_{2}}^{\alpha_{1}} \delta_{\beta_{1}}^{\alpha_{2}} t_{0} \int \frac{d^{d} \mathbf{k}}{(2 \pi)^{d}} \frac{e^{i \mathbf{k} \cdot \mathbf{r}}-1}{|\mathbf{k}|^{2}} \\
& =\delta_{\beta_{2}}^{\alpha_{1}} \delta_{\beta_{1}}^{\alpha_{2}} \frac{t_{0}}{(d-2) S_{d}} \frac{1}{|\mathbf{r}|^{d-2}} \\
& \sim \delta_{\beta_{2}}^{\alpha_{1}} \delta_{\beta_{1}}^{\alpha_{2}} \frac{t}{2 \pi}\left[\frac{1}{\epsilon}-\frac{1}{2} \ln \left(\pi \mu^{2}|\mathbf{r}|^{2} e^{\gamma}\right)\right],
\end{aligned}
$$

where $S_{d}$ is the surface area of the sphere in $d$ dimensions and we have used Eqs. (5.1) and (5.3).

Let us define the renormalized operator

$$
\left[\Omega_{p}\right]\left(\mathbf{r}, \mathbf{r}^{\prime}\right) \equiv Z_{p}^{-2} \Omega_{p}\left(\mathbf{r}, \mathbf{r}^{\prime}\right)
$$

where $Z_{p}$ is the renormalization factor obtained via Eqs. (5.13) and (5.14) for the composite operators defined by Eq. (2.23); all operators belonging to a particular irreducible representation receive the same renormalization in a $\mathrm{NL} \sigma \mathrm{M} .{ }^{59}$

Insisting that $\left\langle\left[\Omega_{p}\right]\left(\mathbf{r}, \mathbf{r}^{\prime}\right)\right\rangle$ is finite (for $\mathbf{r} \neq \mathbf{r}^{\prime}$ ), we see that we must take

$$
\alpha_{p, 1}=n p-\frac{p(p-1)}{2}
$$

in Eq. (5.17). We have obtained the lowest-order expansion coefficient for the group-invariant structure defined by Eqs. (5.16) and (5.17) without explicitly employing group theory, but using only the renormalizability of the NL $\sigma \mathrm{M}$ ! [Basic group-theoretic knowledge was necessary to identify the invariant scaling operators defined by Eq. (2.23), however. $]^{80}$

Finally, we set $t=t^{*}(\epsilon)$ and then we re-exponentiate the expectation of Eq. (5.19) to obtain, at the nontrivial critical point,

$$
\left\langle\left[\Omega_{p}\right]\left(\mathbf{r}, \mathbf{r}^{\prime}\right)\right\rangle \sim \frac{\widetilde{\alpha}_{p}\left(\pi \mu^{2} e^{\gamma}\right)^{-x_{p}^{*}}}{\left|\mathbf{r}-\mathbf{r}^{\prime}\right|^{2 x_{p}^{*}}},
$$

where $x_{p}^{*}$ is the scaling dimension in Eq. (5.14), evaluated at $t=t^{*}$. [ $x_{p}^{p}$ is given explicitly by Eqs. (1.6a), (1.6b), and (1.6c) for the limit $n \rightarrow 0$, appropriate to the MIT.]

Equation (5.21) allows us to define the following "renormalized and normalized" composite operators, which we will enclose with the double square brackets $\llbracket \cdots \rrbracket$. Referring to Eq. (2.23), we designate

$$
\left.\llbracket \mathcal{O}_{p\left[\beta_{1} \ldots \beta_{p}\right]}^{\alpha_{1} \ldots \alpha_{p}} \rrbracket(\mathbf{r}) \equiv Z_{p}^{-1}\left(\pi \mu^{2} e^{\gamma}\right)^{x_{p}^{*} / 2} \mathcal{O}_{p\left[\beta_{1} \ldots \beta_{p}\right]}^{\alpha_{1} \ldots \alpha_{p}}\right](\mathbf{r}) .
$$

Equations (5.19) and (5.21) guarantee that the two-point correlation function between distant operators defined by Eq. (5.22) is both ultraviolet finite and independent of the renormalization scheme.

\section{Operator product expansion at the MIT}

We conclude this section with the construction of the OPE for the operators in Eq. (5.22). At the critical point in $d=2$ $+\epsilon$, the OPE is expected to take the form

$$
\begin{aligned}
& \llbracket \mathcal{O}_{p\left[\beta_{1} \ldots \beta_{p}\right]}^{\alpha_{1} \ldots \alpha_{p}} \rrbracket(\mathbf{r}) \llbracket \mathcal{O}_{p^{\prime}\left[\beta_{1}^{\prime} \ldots \beta_{p^{\prime}}^{\prime}\right]}^{\alpha_{1}^{\prime} \ldots \alpha_{p^{\prime}}^{\prime}} \rrbracket\left(\mathbf{r}^{\prime}\right) \\
& \left.\sim \frac{C_{p, p^{\prime}}^{p+p^{\prime}}}{\left|\mathbf{r}_{d}\right| x_{p}^{*}+x_{p^{\prime}}^{*}-x_{p+p^{\prime}}^{*}} \llbracket \mathcal{O}_{p+p^{\prime}\left[\beta_{1} \ldots \beta_{p} \beta_{1}^{\prime} \ldots \beta_{p^{\prime}}^{\prime},\right.}^{\alpha_{1} \ldots \alpha_{2} \alpha_{1}^{\prime} \ldots \alpha_{p^{\prime}}^{\prime}}\right](\mathbf{R})+\cdots,
\end{aligned}
$$

where $\mathbf{r}_{d} \equiv \mathbf{r}-\mathbf{r}^{\prime}, \mathbf{R} \equiv\left(\mathbf{r}+\mathbf{r}^{\prime}\right) / 2, x_{p}^{*}$ is the scaling dimension defined by Eqs. (5.14), (1.6a), (1.6b), and (1.6c), and $C_{p, p^{\prime}}^{p+p^{\prime}}$ is the (universal) OPE coefficient that we seek, expected to possess an expansion in powers of $t^{*}(\epsilon)$. Equation (5.23) will hold as a replacement rule in the limit $\left|\mathbf{r}_{d}\right| \rightarrow 0$, valid inside correlation functions involving arbitrary configurations of other spatially remote operators. Note that Eq. (5.23) relates a product of maximally antisymmetric operators to a single, maximally antisymmetric operator; the ellipsis "..." on the RHS of this equation represents other, less-relevant operators that are produced in the "fusion" process. We will ignore the contribution of the latter to functional $\mathrm{RG}^{81}$

In order to determine $C_{p, p^{\prime}}^{p+p^{\prime}}$, we compute an arbitrary matrix element $\Gamma_{p+p^{\prime} \gamma_{1} \cdots \gamma_{p+p^{\prime}}}^{\lambda_{1} \ldots \lambda_{p+p^{\prime}}}[\cdots]$ of both sides of Eq. (5.23), as defined by Eq. (5.8). Using Eqs. (5.10) and (5.22) and employing the compact notation introduced above and implemented in Eq. (5.11), the RHS of Eq. (5.23) may be written as

$$
\begin{aligned}
\underline{\Gamma}_{p+p^{\prime}}[\underline{\mathrm{RHS}}]= & Z_{W}^{\left(p+p^{\prime}\right) / 2} Z_{p+p^{\prime}}^{-1}\left(\pi \mu^{2} e^{\gamma}\right)^{x_{p+p^{\prime}}^{*}} \frac{C_{p, p^{\prime}}^{p+p^{\prime}}}{\left|\mathbf{r}_{d}\right|^{x_{p}^{*}+x_{p^{\prime}}^{*} x_{p+p^{\prime}}^{*}}} \\
& \times \underline{\Gamma}_{p+p^{\prime}}^{(0)}\left[\underline{\mathcal{O}}_{p+p^{\prime}}(\mathbf{R})\right],
\end{aligned}
$$


where $Z_{W}\left(Z_{p+p^{\prime}}\right)$ is the field strength (composite operator) renormalization factor and the bare amplitude is given by

$$
\underline{\Gamma}_{p+p^{\prime}}^{(0)}\left[\underline{\mathcal{O}}_{p+p^{\prime}}\right]=\left[1-\frac{1}{2}\left(p+p^{\prime}\right)\left(p+p^{\prime}-1\right) I_{1}\right] \underline{\mathfrak{A}}_{p+p^{\prime}}
$$

In Eq. (5.25), $I_{1}$ is the loop integral defined by Eq. (5.12), where we retain the infrared regularization for now, $h_{0} \neq 0$, while

$$
\underline{\mathfrak{A}}_{p+p^{\prime}} \rightarrow \mathfrak{A}_{p+p^{\prime} \gamma_{1} \ldots \gamma_{p+p^{\prime}} ;\left[\beta_{1} \ldots \beta_{p} \beta_{1}^{\prime} \ldots \beta_{p^{\prime}}^{\prime}\right]}^{\lambda_{1} \ldots \lambda_{p+p^{\prime}} ; \alpha_{1} \ldots \alpha_{p} \alpha_{1}^{\prime} \ldots \alpha_{p^{\prime}}^{\prime}}
$$

denotes the zeroth order amplitude for the matrix element (a pure number) (see Ref. 75 for details).

Similarly, the LHS matrix element of Eq. (5.23) may be written as

$$
\begin{aligned}
\left.\underline{\Gamma}_{p+p^{\prime}} \underline{[\mathrm{LHS}}\right]= & Z_{W}^{\left(p+p^{\prime}\right) / 2} Z_{p}^{-1} Z_{p^{\prime}}^{-1}\left(\pi \mu^{2} e^{\gamma}\right)^{\left(x_{p^{*}}{ }^{*}{ }_{p^{\prime}}{ }^{\prime}\right) / 2} \\
& \times \underline{\Gamma}_{p+p^{\prime}}^{(0)}\left[\underline{\mathcal{O}}_{p}(\mathbf{r}) \underline{\mathcal{O}}_{p^{\prime}}\left(\mathbf{r}^{\prime}\right)\right]
\end{aligned}
$$

where the bare (unrenormalized) amplitude is

$$
\begin{aligned}
\underline{\Gamma}_{p+p^{\prime}}^{(0)} & {\left[\underline{\mathcal{O}}_{p}(\mathbf{r}) \underline{\mathcal{O}}_{p^{\prime}}\left(\mathbf{r}^{\prime}\right)\right] } \\
& \rightarrow\left\langle\left\langle\mathcal{O}_{p\left[\beta_{1} \ldots \beta_{p}\right]}^{\alpha_{1} \ldots \alpha_{p}}(\mathbf{r}) \mathcal{O}_{p^{\prime}\left[\beta_{1}^{\prime} \ldots \beta_{p^{\prime}}^{\prime}\right]}^{\alpha_{1}^{\prime} \ldots \alpha_{p^{\prime}}^{\prime}}\left(\mathbf{r}^{\prime}\right) W_{\gamma_{1}}^{\dagger \lambda_{1}} \cdots W_{\gamma_{p+p^{\prime}}}^{\left.\left.\dagger \lambda_{p+p^{\prime}}\right\rangle\right\rangle}\right.\right.
\end{aligned}
$$

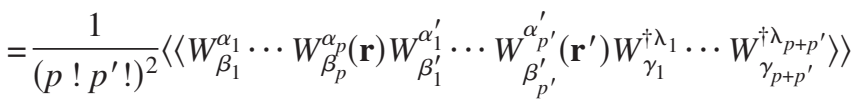

$$
\begin{aligned}
& +\left\{\begin{array}{cc}
\left(p ! p^{\prime} !-1\right) & \text { other terms } \\
\text { obtained by } & \text { permutations }
\end{array}\right\} \text {. }
\end{aligned}
$$

As in Eq. (5.8), the external fields $\left\{\hat{W}^{\dagger}\right\}$ in these equations are assumed to be located far from the vicinity of the operator product $\left(\mathbf{r}\right.$ or $\left.\mathbf{r}^{\prime}\right)$ and from each other, while the doubleangle brackets instruct us to take the one-particle irreducible amplitude, with external legs amputated. ${ }^{60}$

Consider the one-loop renormalization of the term written explicitly in Eq. (5.27b); the other $\left(p ! p^{\prime} !-1\right)$ terms implied in this equation will give identical contributions. The basic renormalization process of an operator product is illustrated in Fig. 4 specifically for the combination

$$
W_{\beta_{1}}^{\alpha_{1}}(\mathbf{r}) \otimes W_{\beta_{2}}^{\alpha_{2}}\left(\mathbf{r}^{\prime}\right) W_{\beta_{3}}^{\alpha_{3}}\left(\mathbf{r}^{\prime}\right)
$$

The vertex $\mathfrak{V}_{4}[$ Fig. 2 and Eq. (5.2)] modifies the operator product in two ways. First, it renormalizes the constituent operators, pairwise permuting indices of legs both tied to either $\mathbf{r}$ or $\mathbf{r}^{\prime}$, as shown in Fig. 4(b) [cf. Fig. 3]. Second, $\mathfrak{V}_{4}$ ties the two operators together by pairwise crosspermuting their indices as depicted in Figs. 4(c) and 4(d).
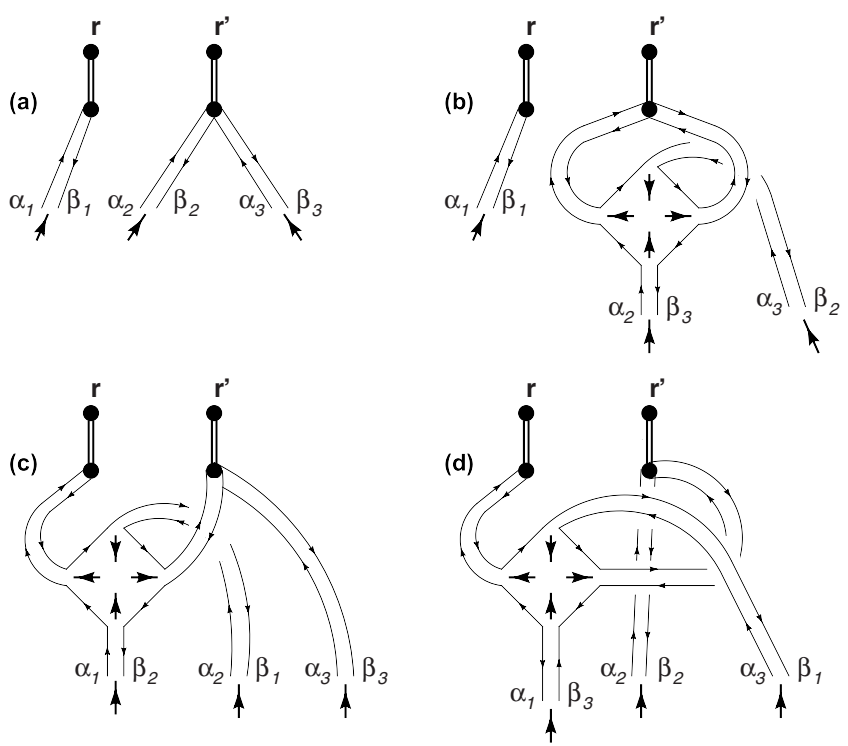

FIG. 4. Basic renormalization process in the OPE.

Now, algebraically, we may express an unsymmetrized product of $q \hat{W}$-field matrix elements $\left(W_{\beta}^{\alpha}\right)$ in terms of the completely antisymmetrized product, native to the most relevant irreducible representation with Casimir parameter $q$ of the NL $\sigma \mathrm{M}$ symmetry group, plus other terms which belong to other (completely symmetric or mixed-symmetry) representations. In particular,

$$
W_{\beta_{1}}^{\alpha_{1}} \cdots W_{\beta_{q}}^{\alpha_{q}}=W_{\left[\beta_{1}\right.}^{\alpha_{1}} \cdots W_{\left.\beta_{q}\right]}^{\alpha_{q}}+\cdots
$$

where again the square brackets $[\cdots]$ denote complete antisymmetrization. The unity coefficient in front of the completely antisymmetrized tensor on the RHS of Eq. (5.28) follows from the fact that the antisymmetrization procedure is a projective operation that kills symmetric or mixedsymmetry terms, but leaves the preantisymmetrized component invariant.

After the vertex acts upon the unsymmetrized operator product displayed explicitly in Eq. (5.27b), giving the appropriate factors for the two types of renormalization depicted in Fig. 4(b) and Figs. 4(c) and 4(d), respectively, we are free to use Eq. (5.28) to replace each resulting unsymmetrized, permuted product with the corresponding completely antisymmetrized version up to less relevant mixed symmetry or higher gradient terms. ${ }^{82}$ The completely antisymmetrized product of $p+p^{\prime}$ factors is just the composite operator on the RHS of the OPE, as defined by Eq. (5.23). Therefore, using the Feynman rules in Eqs. (5.1) and (5.2) and summing all diagram topologies to one loop, Eq. (5.27a) may be written as

$$
\begin{aligned}
& \underline{\Gamma}_{p+p^{\prime}}^{(0)}\left[\underline{\mathcal{O}}_{p}(\mathbf{r}) \underline{\mathcal{O}}_{p^{\prime}}\left(\mathbf{r}^{\prime}\right)\right] \\
& \sim\left(\begin{array}{c}
p+p^{\prime} \\
p
\end{array}\right)\left(\begin{array}{l}
\left.1-I_{1}\left[\frac{p(p-1)}{2}+\frac{p^{\prime}\left(p^{\prime}-1\right)}{2}\right]\right) \\
-I_{2}\left(\mathbf{r}_{d}\right)\left[p p^{\prime}\right]
\end{array}\right) \underline{\mathfrak{A}}_{p+p^{\prime}},
\end{aligned}
$$


where $\underline{\mathfrak{A}}_{p+p^{\prime}}$ denotes the zeroth order matrix element of $\mathcal{O}_{p+p^{\prime}\left[\beta_{1} \ldots \beta_{p} \beta_{1}^{\prime} \ldots \beta_{p^{\prime}}^{\prime}\right]}^{\alpha_{1} \ldots \alpha_{p} \alpha_{1}^{\prime} \ldots \alpha_{p^{\prime}}^{\prime}}$, as in Eq. (5.25), $I_{1}$ is the integral defined by Eq. (5.12), and

$$
I_{2}\left(\mathbf{r}_{d}\right)=\frac{-t_{0}}{2} \int \frac{d^{d} \mathbf{k}}{(2 \pi)^{d}} \frac{e^{i \mathbf{k} \cdot \mathbf{r}_{d}}}{|\mathbf{k}|^{2}+h_{0} t_{0}}
$$

Let us briefly comment upon the origin of the various combinatoric factors in Eq. (5.29). The prefactor

$$
\left(\begin{array}{c}
p+p^{\prime} \\
p
\end{array}\right)
$$

originates from the normalization convention used in Eq. (2.23). The factors of $p(p-1) / 2$ and $p^{\prime}\left(p^{\prime}-1\right) / 2$ count the number of inequivalent ways leg indices associated with either operator $\underline{\mathcal{O}}_{p}$ or $\mathcal{O}_{p^{\prime}}$ (but not both) may be permuted, as occurred previously in the scaling dimension calculation [Eq. (5.10)]. The factor of $p p^{\prime}$ counts the number of inequivalent ways one leg index from each operator may be interpermuted, as in Figs. 4(c) and 4(d).

Equating the left-hand and right-hand sides of the OPE, Eqs. (5.24)-(5.26) and (5.29), and expanding everything to the lowest nontrivial order in $t$, we obtain

$$
C_{p, p^{\prime}}^{p+p^{\prime}} \sim\left(\begin{array}{c}
p+p^{\prime} \\
p
\end{array}\right)\left(\begin{array}{c}
1+p p^{\prime}\left[I_{1}-I_{2}\left(\mathbf{r}_{d}\right)\right]+\ln \left[\frac{Z_{p+p^{\prime}}}{Z_{p} Z_{p^{\prime}}}\right] \\
-\frac{1}{2}\left(x_{p+p^{\prime}}^{*}-x_{p}^{*}-x_{p^{\prime}}^{*}\right) \\
\times \ln \left(\pi \mu^{2}\left|\mathbf{r}_{d}\right|^{2} e^{\gamma}\right)
\end{array}\right) .
$$

We may now take the limit $h_{0} \rightarrow 0$ because the combination $2\left[I_{1}-I_{2}\left(\mathbf{r}_{d}\right)\right][$ Eqs. (5.12) and (5.30)] gives the IR-finite integral evaluated previously in Eq. (5.18), above. Using Eq. (5.14), Eq. (5.31) simplifies to the expression

$$
C_{p, p^{\prime}}^{p+p^{\prime}} \sim\left(\begin{array}{c}
p+p^{\prime} \\
p
\end{array}\right)\left(\begin{array}{c}
1-\frac{1}{2}\left(x_{p+p^{\prime}}^{*}-x_{p}^{*}-x_{p^{\prime}}^{*}+t \frac{p p^{\prime}}{4 \pi}\right) \\
\times \ln \left(\pi \mu^{2}\left|\mathbf{r}_{d}\right|^{2} e^{\gamma}\right)
\end{array}\right) .
$$

In general, the RHS of this equation is an ultraviolet (UV)finite, nonzero function of the operator separation $\mathbf{r}_{d}$. At the nontrivial critical point $t=t^{*}$ in $d=2+\epsilon$, however, we have [from Eq. (5.14)]

$$
C_{p, p^{\prime}}^{p+p^{\prime}} \sim\left(\begin{array}{c}
p+p^{\prime} \\
p
\end{array}\right)+\boldsymbol{O}\left(t^{* 2}\right) .
$$

At the Anderson metal-insulator transition $(n \rightarrow 0), t^{*}$ $=4 \pi \sqrt{2 \epsilon}+\boldsymbol{O}(\epsilon)$. Thus the OPE coefficient for the operators with normalization determined by Eq. (5.22) is independent of $\sqrt{\epsilon}$ to order $\epsilon^{83}$

The result in Eq. (5.33) should be contrasted with a similar computation in $\phi^{4}$ theory in $d=4-\epsilon$ : at the Wilson-Fisher fixed point, the fusion of two elementary renormalized and normalized $\llbracket \phi \rrbracket$ fields into the mass operator $\llbracket \phi^{2} \rrbracket$ yields an OPE coefficient that acquires a correction at the lowest nontrivial order in the quartic coupling strength $\lambda^{*} \propto \boldsymbol{\epsilon}+\boldsymbol{O}\left(\boldsymbol{\epsilon}^{2}\right)$ (see, e.g., Ref. 84). As in the above NL $\sigma \mathrm{M}$ calculation, the normalization of the operators $\llbracket \phi \rrbracket$ and $\llbracket \phi^{2} \rrbracket$ is chosen so as to give two-point autocorrelation functions independent of the renormalization scheme.

Finally, tracing over pairs of indices in Eq. (5.23) allows the OPE to be written as

$$
\llbracket \mathcal{O}_{p} \rrbracket(\mathbf{r}) \llbracket \mathcal{O}_{p^{\prime}} \rrbracket\left(\mathbf{r}^{\prime}\right) \sim \frac{C_{p, p^{\prime}}^{p+p^{\prime}}}{\left|\mathbf{r}_{d}\right|_{p^{*}+x_{p^{\prime}}^{*}{ }^{*} x_{p+p^{\prime}}^{*}}^{*}} \llbracket \mathcal{O}_{p+p^{\prime}} \rrbracket(\mathbf{R})+\cdots,
$$

where

$$
\llbracket \mathcal{O}_{p} \rrbracket(\mathbf{r}) \equiv \sum_{\alpha_{1}=1}^{n} \ldots \sum_{\alpha_{p}=1}^{n} \llbracket \mathcal{O}_{p\left[\alpha_{1} \ldots \alpha_{p}\right]}^{\alpha_{1} \ldots \alpha_{p}} \rrbracket(\mathbf{r})=Z_{p}^{-1}\left(\pi \mu^{2} e^{\gamma}\right)^{x_{p}^{*} / 2} \mathcal{O}_{p}(\mathbf{r})
$$

is the renormalized and normalized version of the bare operator defined by Eq. (2.24).

The OPE in Eq. (5.34), together with the coefficient $C_{p, p^{\prime}}^{p+p^{\prime}}$ given by Eq. (5.33), constitutes the primary technical result of this paper. We have succeeded in associating a unique, properly normalized operator $\llbracket \mathcal{O}_{p} \rrbracket(\mathbf{r})$ to the $p$ th moment of the LDOS and demonstrated that the family of such operators obeys the OPE set forth in Eq. (1.11) in Sec. I. This is the necessary input to the functional RG scheme used in Sec. III to extract the typical multifractal spectrum $\tau(q)$ in Eq. (3.25).

\section{ACKNOWLEDGMENTS}

We would like to thank Igor Aleiner, Boris Altshuler, and Gabriel Kotliar for helpful discussions and probing questions. This work was supported in part by the Nanoscale Science and Engineering Initiative of the National Science Foundation under NSF Award No. CHE-06-41523 and by the New York State Office of Science, Technology, and Academic Research (NYSTAR) (M.S.F.). This work was also supported in part by the NSF under Grants No. DMR0547769 (M.S.F.), No. PHY05-51164 (S.R.), and No. DMR0706140 (A.W.W.L.). S.R. thanks the Center for Condensed Matter Theory at University of California, Berkeley for its support. 
*psiborf@rci.rutgers.edu

${ }^{1}$ For a review, see, e.g., P. A. Lee and T. V. Ramakrishnan, Rev. Mod. Phys. 57, 287 (1985); B. L. Altshuler and B. D. Simons, in Proceedings of the Les Houches Summer School on Mesoscopic Quantum Physics, edited by E. Akkermans, G. Montambaux, J. L. Pichard, and J. Zinn-Justin (Elsevier, Amsterdam, 1995).

${ }^{2}$ A. D. Mirlin, Phys. Rep. 326, 259 (2000); Proceedings of the International School of Physics "Enrico Fermi," Course CXLIII, edited by G. Casati, I. Guarneri, and U. Smilansky (IOS Press, Amsterdam, 2000), pp. 223-298.

${ }^{3}$ For a recent review, see, e.g., F. Evers and A. D. Mirlin, Rev. Mod. Phys. 80, 1355 (2008).

${ }^{4}$ For a review, see M. Janssen, Int. J. Mod. Phys. B 8, 943 (1994).

${ }^{5}$ For a review, see B. Huckestein, Rev. Mod. Phys. 67, 357 (1995).

${ }^{6} \mathrm{~K}$. Efetov, Supersymmetry in Disorder and Chaos (Cambridge University Press, Cambridge, England, 1997).

${ }^{7}$ The notion of the multifractality was recently extended to the wave-function statistics at surfaces or boundaries ("surface multifractality") of a bulk disordered electronic system at criticality. A. R. Subramaniam, I. A. Gruzberg, A. W. W. Ludwig, F. Evers, A. Mildenberger, and A. D. Mirlin, Phys. Rev. Lett. 96, 126802 (2006).

${ }^{8}$ F. Wegner, Z. Phys. B 36, 209 (1980); in Localization and Metal Insulator Transitions, edited by H. Fritzsche and D. Adler, Institute for Amorphous Studies Series (Plenum Press, New York, 1985).

${ }^{9}$ C. Castellani and L. Peliti, J. Phys. A 19, L429 (1986).

${ }^{10}$ T. C. Halsey, M. H. Jensen, L. P. Kadanoff, I. Procaccia, and B. I. Shraiman, Phys. Rev. A 33, 1141 (1986).

${ }^{11}$ A. Kudrolli, V. Kidambi, and S. Sridhar, Phys. Rev. Lett. 75, 822 (1995).

${ }^{12}$ Y. V. Fyodorov and A. D. Mirlin, Phys. Rev. B 51, 13403 (1995).

${ }^{13}$ K. Hashimoto, C. Sohrmann, J. Wiebe, T. Inaoka, F. Meier, Y. Hirayama, R. A. Römer, R. Wiesendanger, and M. Morgenstern, Phys. Rev. Lett. 101, 256802 (2008).

${ }^{14}$ M. Schreiber and H. Grussbach, Phys. Rev. Lett. 67, 607 (1991).

${ }^{15}$ W. Pook and M. Janssen, Z. Phys. B: Condens. Matter 82, 295 (1991).

${ }^{16}$ F. Evers and A. D. Mirlin, Phys. Rev. Lett. 84, 3690 (2000).

${ }^{17}$ F. Evers, A. Mildenberger, and A. D. Mirlin, Phys. Rev. B 64, 241303 (2001).

${ }^{18}$ A. Mildenberger, F. Evers, and A. D. Mirlin, Phys. Rev. B 66, 033109 (2002).

${ }^{19}$ H. Obuse, A. R. Subramaniam, A. Furusaki, I. A. Gruzberg, and A. W. W. Ludwig, Phys. Rev. Lett. 101, 116802 (2008).

${ }^{20}$ F. Evers, A. Mildenberger, and A. D. Mirlin, Phys. Rev. Lett. 101, 116803 (2008).

${ }^{21}$ L. J. Vasquez, A. Rodriguez, and R. A. Römer, Phys. Rev. B 78, 195106 (2008).

${ }^{22}$ A. Rodriguez, L. J. Vasquez, and R. A. Römer, Phys. Rev. B 78, 195107 (2008).

${ }^{23}$ A. Rodriguez, L. J. Vasquez, and R. A. Römer, Phys. Rev. Lett. 102, 106406 (2009).

${ }^{24}$ C. C. Chamon, C. Mudry, and X.-G. Wen, Phys. Rev. Lett. 77, 4194 (1996).

${ }^{25}$ A. D. Mirlin and F. Evers, Phys. Rev. B 62, 7920 (2000).

${ }^{26}$ C. Mudry, C. Chamon, and X.-G. Wen, Nucl. Phys. B 466, 383
(1996).

${ }^{27}$ I. I. Kogan, C. Mudry, and A. M. Tsvelik, Phys. Rev. Lett. 77, 707 (1996).

${ }^{28}$ D. Carpentier and P. Le Doussal, Nucl. Phys. B 588, 565 (2000).

${ }^{29}$ D. Carpentier and P. Le Doussal, Phys. Rev. E 63, 026110 (2001).

${ }^{30}$ C. Mudry, S. Ryu, and A. Furusaki, Phys. Rev. B 67, 064202 (2003).

${ }^{31}$ V. N. Prigodin and B. L. Altshuler, Phys. Rev. Lett. 80, 1944 (1998).

${ }^{32} \mathrm{An}$ analogous situation occurs for the random-energy model (REM) (Ref. 85), where the normalized partition function $Z(\beta) /[Z(1)]^{\beta}$ plays the role of $P_{q}$ [Eq. (1.1)] in the present work, with the identification $\beta \leftrightarrow q ; \beta$ denotes the inverse temperature in the REM. (A generalized REM describes delocalization in the 2D chiral Dirac model studied in Refs. 24, 26-30, and 37-41; the connection is articulated in Refs. 24 and 29.) At high temperatures, $Z(\beta)$ is a self-averaging quantity with narrow statistics, though arbitrarily large moments of $Z(\beta)$ for any $\beta>0$ suggest log-normal asymptotics (Ref. 85). For $\beta>\beta_{c}, Z(\beta)$ becomes broadly distributed (Refs. 24 and 85 ). By contrast, the free energy $F=-T \ln Z$, in analogy with the typical $\tau(q)$ spectrum, remains narrowly distributed for all $\beta$.

${ }^{33}$ The averaged spectra $\widetilde{\tau}(q)$ defined in Eq. (1.3b) may exhibit a further termination arising from the condition $\alpha \geq 0$. See, e.g., Ref. 3 and H. Obuse, A. R. Subramaniam, A. Furusaki, I. A. Gruzberg, and A. W. W. Ludwig, Physica E (Amsterdam) 40, 1404 (2008).

${ }^{34}$ A. M. M. Pruisken, Phys. Rev. B 31, 416 (1985).

${ }^{35}$ D. Höf and F. Wegner, Nucl. Phys. B 275, 561 (1986).

${ }^{36}$ F. Wegner, Nucl. Phys. B 280, 193 (1987); 280, 210 (1987).

${ }^{37}$ A. W. W. Ludwig, M. P. A. Fisher, R. Shankar, and G. Grinstein, Phys. Rev. B 50, 7526 (1994).

${ }^{38}$ H. E. Castillo, C. C. Chamon, E. Fradkin, P. M. Goldbart, and C. Mudry, Phys. Rev. B 56, 10668 (1997).

${ }^{39}$ O. Motrunich, K. Damle, and D. A. Huse, Phys. Rev. B 65, 064206 (2002).

${ }^{40}$ T. Fukui, Phys. Rev. B 68, 153307 (2003).

${ }^{41}$ H. Yamada and T. Fukui, Nucl. Phys. B 679, 632 (2004).

${ }^{42}$ Luca Dell'Anna, Nucl. Phys. B 750, 213 (2006).

${ }^{43}$ F. Wegner, Z. Phys. B 35, 207 (1979).

${ }^{44}$ B. L. Altshuler, V. E. Kravtsov, and I. V. Lerner, Pis'ma Zh. Eksp. Teor. Fiz. 43, 342 (1986) [JETP Lett. 43, 441 (1986)]; Zh. Eksp. Teor. Fiz. 91, 2276 (1986) [Sov. Phys. JETP 64, 1352 (1986)]; Phys. Lett. A 134, 488 (1989).

${ }^{45}$ B. L. Altshuler, V. E. Kravtsov, and I. V. Lerner, in Mesoscopic Phenomena in Solids, edited by B. L. Altshuler, P. A. Lee, and R. A. Webb (North-Holland, Amsterdam, 1991), Vol. 449.

${ }^{46}$ F. Wegner, Z. Phys. B: Condens. Matter 44, 9 (1981).

${ }^{47}$ A. J. McKane and M. Stone, Ann. Phys. (N.Y.) 131, 36 (1981).

${ }^{48}$ In contrast, among the nonstandard classes (Ref. 55) extensively studied in the recent past, including the 2D Dirac models (Refs. 24, 26-30, and 37-41) that inspired the present work, the average density of states may alternatively vanish or diverge at the delocalization transition, implying that $x_{1}^{*} \neq 0$. Equation (1.5) applies equally to this more general case.

${ }^{49}$ Although the results for the symplectic symmetric class are also known, they have no direct physical application in Anderson localization. There is no perturbatively accessible, infrared nontrivial fixed point in the symplectic symmetry class once the replica limit is taken. 
${ }^{50}$ B. A. Muzykantskii and D. E. Khmelnitskii, Phys. Rev. B 51, 5480 (1995).

${ }^{51}$ V. I. Fal'ko and K. B. Efetov, Europhys. Lett. 32, 627 (1995); Phys. Rev. B 52, 17413 (1995).

${ }^{52}$ A. D. Mirlin, Phys. Rev. B 53, 1186 (1996).

${ }^{53}$ The IPR $P_{q}$ defined by Eq. (1.1) can be discretized using the following construction: the sample of size $L^{d}$ is partitioned into $N$ boxes each of size $a^{d}$, with $a \ll L$. Let $\Omega_{i}$ denote the $i$ th box. Then one defines the box probability $\mu_{i} \equiv \int_{\Omega_{i}} d^{d} \mathbf{r}\left|\psi_{i}(\mathbf{r})\right|^{2}$, where we obviously have $\Sigma_{i} \mu_{i}=1$. The IPR is expressed as $P_{q} \sim \sum_{i} \mu_{i}^{q}$, which gives the same scaling behavior as Eq. (1.1) in the limit that $N \rightarrow \infty$ for $q \geq 0$. Moreover, the IPR defined through the box probabilities extends the multifractal spectrum $\tau(q)$ to negative $q$.

${ }^{54}$ B. Duplantier and A. W. W. Ludwig, Phys. Rev. Lett. 66, 247 (1991).

${ }^{55}$ M. R. Zirnbauer, J. Math. Phys. 37, 4986 (1996); D. Bernard and A. LeClair, J. Phys. A 35, 2555 (2002); A. Altland, B. D. Simons, and M. R. Zirnbauer, Phys. Rep. 359, 283 (2002).

${ }^{56}$ A. Kolmogorov, I. Petrovsky, and N. Piscounov, Moscow Univ. Math. Bull. (Engl. Transl.) 1, 1 (1937).

${ }^{57}$ K. B. Efetov, A. I. Larkin, and D. E. Khmel'nitskii, Zh. Eksp. Teor. Fiz. 79, 1120 (1980) [Sov. Phys. JETP 52, 568 (1980)].

${ }^{58}$ A. M. Polyakov, Phys. Lett. 59B, 79 (1975).

${ }^{59}$ See, e.g., E. Brézin, J. Zinn-Justin, and J. C. Le Guillou, Phys. Rev. D 14, 2615 (1976); Phys. Rev. B 14, 4976 (1976).

${ }^{60}$ See, e.g., J. Zinn-Justin, Quantum Field Theory and Critical Phenomena, 4th ed. (Oxford University Press, New York, 2002).

${ }^{61}$ The key to Eq. (2.16) is the standard angular-momentum addition formula

$$
\begin{aligned}
& Y_{l_{1}, m_{1}}\left(\pi_{+}, \pi_{-}, \sigma\right) Y_{l_{2}, m_{2}}\left(\pi_{+}, \pi_{-}, \sigma\right) \\
& \quad=\sum_{l, m}\left\langle l_{1} l_{2} ; \operatorname{lm} \mid l_{1} l_{2} ; m_{1} m_{2}\right\rangle c_{l_{1}, l_{2}}^{l} Y_{l, m}\left(\pi_{+}, \pi_{-}, \sigma\right),
\end{aligned}
$$

where $\left\langle l_{1} l_{2} ; \operatorname{lm} \mid l_{1} l_{2} ; m_{1} m_{2}\right\rangle$ is a Clebsch-Gordan coefficient and $c_{l_{1}, l_{2}}^{l}$ is a normalization constant that is independent of $\left\{m, m_{1}, m_{2}\right\}$. As an example, one has $\left(\pi_{+}+\pi_{-}\right)^{2}=\frac{1}{\lambda_{2}}\left[Y_{2,2}\right.$ $\left.-\sqrt{\frac{2}{3}} Y_{2,0}+Y_{2,-2}\right]+\frac{1}{\lambda_{0}} \frac{4}{3} Y_{0,0}$, where the coefficients $\left\{\lambda_{2}, \lambda_{0}\right\}$ are determined by Eq. (2.18).

${ }^{62}$ Note, however, that for the $O(3) / O(2)$ model, higher $l$ operators possess less relevant scaling dimensions at the nontrivial critical point in $d=2+\epsilon$ (Ref. 59). The existence of operators with arbitrarily negative scaling dimensions in the unitary class NL $\sigma \mathrm{M}$ emerges due to the replica $n \rightarrow 0$ limit.

${ }^{63}$ Because of the symmetry restoration linking the $\pi\left(\hat{W}, \hat{W}^{\dagger}\right)$ and $\sigma\left(\sqrt{\hat{\mathbb{I}}_{n}-\hat{W} \hat{W}^{\dagger}}, \sqrt{\hat{\mathbb{I}}_{n}-\hat{W}^{\dagger} \hat{W}}\right)$ NL $\sigma \mathrm{M}$ fields at the nontrivial critical point in $d=2+\epsilon$ (for fixed $n \in\{1,2, \ldots\}$ ), many other eigenoperators exist that share the same "maximally" relevant eigenvalues as those defined by Eqs. (2.23) and (2.24). Our choice to focus on the latter operator family is one of convenience.

${ }^{64}$ J. Cardy, Scaling and Renormalization in Statistical Physics (Cambridge University Press, Cambridge, England, 1996); J. Cardy, Proceeding of the Les Houches School of Theoretical Physics, XLIX, 1988, edited by E. Brézin and J. Zinn-Justin (North-Holland, Amsterdam, 1988); A. W. W. Ludwig and J. L. Cardy, Nucl. Phys. B 285, 687 (1987).
${ }^{65}$ In principle, we could execute a straightforward (e.g. Wilsonian) renormalization-group program on the theory defined by Eqs. (2.7) and (2.27). In fact, a closely related calculation was originally performed long ago, before the invention of the FRG, by Altshuler et al. (Refs. 44 and 45) within the context of mesoscopic fluctuations. These authors studied the TRI orthogonal class and their calculation was complicated by the fact that they did not employ a basis of eigenoperators, such as those defined by Eq. (2.23) for the (different, broken TRI) unitary class; more importantly, they neglected the nonlinear coupling between different moments implied by the OPE, Eq. (1.11). It seems that a Wilsonian RG approach, such as that implemented in Ref. 45, does not produce as easily the features that we seek (such as, e.g. the lowest-order fusion process $\mathcal{O}_{1} \otimes \mathcal{O}_{1} \rightarrow \mathcal{O}_{2}$, as represented by a term $\propto Y_{1}^{2}$ in the one-loop beta function for $Y_{2}$ ). Rather than using the FRG within the Wilsonian scheme, we deploy here the field theory approach explicated in Sec. V.

${ }^{66}$ The normalization of $\llbracket \mathcal{O}_{p q} \rrbracket$ is chosen to depend only upon the product of $p$ and $q$ and is proportional to $1 /(p q)$ ! [see Eq. (5.35) in the technical Sec. V of this paper]. The explicit factor $(p q) ! / p$ ! in Eq. (3.1) effectively resets this (RG schemeindependent part of) the operator normalization to $1 / p$ !, independent of $q$, which is more convenient for the FRG argument that follows.

${ }^{67}$ A multilocal field theory, which can directly treat the probability distribution of the Green's functions and the conductance, was proposed by Yudson in Ref. 86.

${ }^{68}$ R. Abou-Chacra, P. W. Anderson, and D. J. Thouless, J. Phys. C 6, 1734 (1973); R. Abou-Chacra and D. J. Thouless, ibid. 7, 65 (1974).

${ }^{69}$ A. D. Mirlin and Y. V. Fyodorov, Phys. Rev. Lett. 72, 526 (1994); V. Dobrosavljević and G. Kotliar, ibid. 78, 3943 (1997).

${ }^{70}$ A. D. Mirlin and Y. V. Fyodorov, J. Phys. I 4, 655 (1994).

${ }^{71}$ Y. V. Fyodorov, Pis'ma Zh. Eksp. Teor. Fiz. 78, 286 (2003) [JETP Lett. 78, 250 (2003)].

${ }^{72}$ C. Castellani, C. Di Castro, and L. Peliti, J. Phys. A 19, L1099 (1986).

${ }^{73}$ E. Brézin, S. Hikami, and J. Zinn-Justin, Nucl. Phys. B 165, 528 (1980).

${ }^{74}$ Strictly speaking, the (bare, unrenormalized) matrix element in Eq. (5.8) is a mixed position and momentum space object, the precise definition of which is given by the correlation function

$$
\begin{aligned}
& \Gamma_{p \gamma_{1} \ldots \gamma_{p}}^{(0) \lambda_{1} \ldots \lambda_{p}}\left[\mathcal{O}_{p\left[\beta_{1} \ldots \beta_{p}\right]}^{\alpha_{1} \ldots \alpha_{p}}(\mathbf{r})\right] \\
& \quad=\left\langle\mathcal{O}_{p\left[\beta_{1} \ldots \beta_{p}\right]}^{\alpha_{1} \ldots \alpha_{p}}(\mathbf{r}) W_{\gamma_{1}}^{\dagger \lambda_{1}}\left(\mathbf{k}_{1}\right) \cdots W_{\gamma_{p}}^{\dagger \lambda_{p}}\left(\mathbf{k}_{p}\right)\right\rangle / G\left(\mathbf{k}_{1}\right) G\left(\mathbf{k}_{2}\right) \cdots G\left(\mathbf{k}_{p}\right),
\end{aligned}
$$

where $G(\mathbf{k})$ is the "one-particle" propagator, which has the amplitude given by Eq. (5.1) at tree level. Only completely connected diagrams are counted in the evaluation of the matrix element. The external momenta $\left\{\mathbf{k}_{1}, \ldots, \mathbf{k}_{p}\right\}$ can be taken to vanish in this equation.

${ }^{75}$ The tree level amplitude $\mathfrak{A}_{p \gamma_{1} \ldots \gamma_{p} ;\left[\beta_{1} \ldots \beta_{p}\right]}^{\lambda_{1} \ldots \alpha_{1}, \ldots \alpha_{p}}$ can be written as a sum of $2 p$-fold products of Kronecker delta functions. In the case of the operator $\mathcal{O}_{2\left[\beta_{1} \beta_{2}\right]}^{\alpha_{1} \alpha_{2}}(\mathbf{r})=(1 / 2 !)^{2}\left[W_{\beta_{1}}^{\alpha_{1}} W_{\beta_{2}}^{\alpha_{2}}(\mathbf{r})-W_{\beta_{2}}^{\alpha_{1}} W_{\beta_{1}}^{\alpha_{2}}(\mathbf{r})\right]$, one finds the amplitude

$$
\begin{aligned}
\mathfrak{A}_{\left.2 \gamma_{1} \gamma_{2} ; \beta_{1} \beta_{2}\right]}^{\lambda_{1} \lambda_{2} ; \alpha_{1} \alpha_{2}}= & (1 / 2 !)^{2}\left[\delta_{\beta_{1}}^{\lambda_{1}} \delta_{\gamma_{1}}^{\alpha_{1}} \delta_{\beta_{2}}^{\lambda_{2}} \delta_{\gamma_{2}}^{\alpha_{2}}+\delta_{\beta_{2}}^{\lambda_{1}} \delta_{\gamma_{1}}^{\alpha_{2}} \delta_{\beta_{1}}^{\lambda_{2}} \delta_{\gamma_{2}}^{\alpha_{1}}\right. \\
& \left.-\delta_{\beta_{2}}^{\lambda_{1}} \delta_{\gamma_{1}}^{\alpha_{1}} \delta_{\beta_{1}}^{\lambda_{2}} \delta_{\gamma_{2}}^{\alpha_{2}}-\delta_{\beta_{1}}^{\lambda_{1}} \delta_{\gamma_{1}}^{\alpha_{2}} \delta_{\beta_{2}}^{\lambda_{2}} \delta_{\gamma_{2}}^{\alpha_{1}}\right] .
\end{aligned}
$$


${ }^{76}$ S. Elitzur, IAS preprint, 1979 (unpublished).

${ }^{77}$ A. McKane and M. Stone, Nucl. Phys. B 163, 169 (1980).

${ }^{78}$ D. J. Amit and G. B. Kotliar, Nucl. Phys. B 170, 187 (1980).

${ }^{79}$ As discussed in Ref. 78 , the basic problem is that noninvariant correlation functions suffer UV divergences for $d \geq 2$ and IR divergences for $d \leq 2$ - there is no finite window of dimensionality free from both UV and IR problems. Within dimensional regularization, noncovariant correlators cannot be regularized via analytical continuation in $d$, so renormalized PT does not exist for these objects. By contrast, invariant correlators can be shown to be IR and UV finite for $0<d<2$; in this case, dimensional regularization works in the usual way.

${ }^{80} \mathrm{As}$ an amusing aside, the same technique employed above can be used to derive the Gegenbauer polynomials, expanded in terms of $\vec{\pi}$ and $\sigma=1-\vec{\pi} \cdot \vec{\pi} / 2+\cdots$, in the $O(n) / O(n-1)$ model, given a knowledge only of the one-loop scaling dimension for composite eigenoperators belonging to a particular irreducible representation.

${ }^{81}$ Note that in order for the most relevant process in Eq. (5.23) to occur, it is necessary that all of the lower indices and all of the upper indices appearing on the LHS of this equation separately differ (i.e., no upper indices have the same value and no lower indices have the same value, but upper indices may take the same values as lower ones). Otherwise, the right-hand side of this equation vanishes, up to less relevant contributions.

${ }^{82}$ We neglect higher gradient operators throughout this paper, which are important to the computation of conductance, rather than LDOS fluctuations (Refs. 44, 45, and 87).

${ }^{83}$ Note that the OPE coefficient [Eq. (5.33)] and hence the coupled RG flow equations obtained in Sec. III [Eq. (3.6)] are similar to those obtained for the chiral random vector potential and class $B D I$ 2D Dirac fermion models (Ref. 30). Indeed, the only qualitative difference is that the average of the LDOS acquires a relevant anomalous dimension in these chiral class models (Refs. 30, 37, and 39) whereas the unitary class is characterized by a noncritical average LDOS, which is the consequence of the Ward identity [see also the discussion following Eqs. (1.5)-(1.8b)].

${ }^{84}$ L. S. Brown, Quantum Field Theory (Cambridge University Press, New York, 1992).

${ }^{85}$ B. Derrida, Phys. Rev. Lett. 45, 79 (1980); Phys. Rev. B 24, 2613 (1981).

${ }^{86}$ V. I. Yudson, Phys. Rev. Lett. 94, 156601 (2005).

${ }^{87}$ F. Wegner, Nucl. Phys. B 354, 441 (1991). 